\title{
Coupling damage and plasticity for a phase-field regularisation of brittle, cohesive and ductile fracture: one-dimensional examples
}

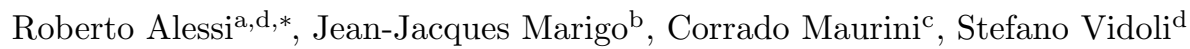 \\ ${ }^{a}$ Dipartimento di Matematica, Università di Roma La Sapienza, Piazzale Aldo Moro 5, 00185 Roma IT \\ ${ }^{b}$ Laboratoire de Mécanique des Solides, Ecole Polytechnique, 91128 Palaiseau Cedex, France \\ 'Sorbonne Universités, UPMC Univ Paris 06, CNRS, UMR 7190, Institut Jean Le Rond d'Alembert, F-75005 Paris, France \\ ${ }^{d}$ Dipartimento di Ingegneria Strutturale e Geotecnica, Università di Roma La Sapienza, Via Eudossiana 18, 00184 Roma IT
}

\begin{abstract}
Plasticity and damage are two fundamental phenomena in nonlinear solid mechanics associated to the development of inelastic deformations and the reduction of the material stiffness. Alessi et al. [5] have recently shown, through a variational framework, that coupling a gradient-damage model with plasticity can lead to macroscopic behaviours assimilable to ductile and cohesive fracture. Here, we further expand this approach considering specific constitutive functions frequently used in phase-field models of brittle fracture. A numerical solution technique of the coupled elastodamage-plasticity problem, based on an alternate minimisation algorithm, is proposed and tested against semi-analytical results. Considering a one-dimensional traction test, we illustrate the properties of four different regimes obtained by a suitable tuning of few key constitutive parameters. Namely, depending on the relative yield stresses and softening behaviours of the plasticity and the damage criteria, we obtain macroscopic responses assimilable to (i) brittle fracture à la Griffith, (ii) cohesive fractures of the Barenblatt or Dugdale type, and (iii) a sort of cohesive fracture including a depinning energy contribution. The comparisons between numerical and analytical results prove the accuracy of the proposed numerical approaches in the considered quasi-static time-discrete setting, but they also emphasise some subtle issues occurring during time-discontinuous evolutions.
\end{abstract}

Keywords: Variational methods, Phase-field, Cohesive fracture, Ductile fracture

\section{Introduction}

Variational approaches have been proved to be useful tools to formulate mathematically sound models of defect mechanics, understand their properties, and devise effective numerical solution strategies. The variational approach of brittle fracture and the associated regularised formulations [23, 22, 24, 40] are nowadays widely adopted in computational mechanics. The mechanical interpretation of the regularised models as damage models added a further insight on the prediction of crack nucleation in the quasi-brittle setting, giving a precise connection between the regularising length-scale and the maximum allowable stress before failure [57, 25].

While the initial works focused on brittle materials, more recently several authors attempted to extend the variational approach to ductile failure. The general idea is to introduce plastic strains and suitable evolution laws, for coupling damage to plasticity, revisiting in a variational phase-field framework the classical approaches to ductile failure [18, 19, 43, 65, 63. A phase-field (damage) fracture model coupled with plasticity has been proposed by [10,

\footnotetext{
${ }^{*}$ Corresponding author

$U R L$ : roberto.alessi@uniroma1.it (Roberto Alessi)
}

with a phenomenological approach, and by [52] in a variational setting. These works have been subsequently extended to include geometric nonlinearities in [11, 53, 20. Further insights on the role of the constitutive parameters on the crack path are given in 46. Imposing an intrinsic link between damage and plastic dissipation, [12, 41] proposed a phase-field model of plastic slip-lines. References [47, 48] discussed how non-local softening plasticity models can be used to account for macroscopic cracks.

A more general class of models for coupling damage and plasticity has been presented and analysed in [5, 6. These works introduce the regularisation only on the damage variable, through a gradient term penalised by an internal material length, while keeping plasticity local and allowing the development of sharp plastic localisations. The discussion of the role of the constitutive assumptions on the emerging macroscopic behaviours shows that damage and plastic localisations may interact to produce cohesive-like cracks. This result has been confirmed through rigorous asymptotic analysis in 30, 31, 34, showing the convergence of a special case of the model presented in [5] to a cohesive sharp crack model. References [29] and [39] recently proposed an alternative interesting phase-field model of cohesive fracture without introducing plastic effects.

The aim of this paper is to extend the analysis of the 
variational model presented in 5]. Therein, an ad-hoc choice of the constitutive laws was used to obtain closed form solutions. Here, we perform the analysis with the constitutive functions classically used in phase-field models of fracture and focus on the numerical solution of the evolution problem. Through the comparison with semianalytical results, we provide several insights on the features and limitations of a staggered numerical scheme for the solution of the quasi-static evolution problem. We discuss, in particular, the effect of snap-back phenomena and irreversibility conditions in the numerical response. We show that one can separately tune the softening behaviour of the yield criteria for damage and plasticity through the choice of the stiffness-degradation and plastic yield functions. Correspondingly, several macroscopic behaviours are obtained, including purely brittle, ductile, and several types of cohesive responses. The results are resumed in Figure 1, which reports four possible dependencies of the fracture dissipated energy $\mathcal{D}_{\mathrm{f}}$ and the stress $\sigma$ on the

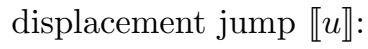

- Griffith's brittle fracture (gray curve), 42, where the dissipated energy is independent of the crack opening.

- Barenblatt's cohesive fracture (blue curve), 17, where the stress across the crack decreases monotonically with the displacement jump, never attaining zero.

- Dugdale-like cohesive fracture (red curve) where the stress across the crack decreases monotonically with the displacement jump and attains zero at a finite value, $u_{\mathrm{c}}$. The original Dugdale's model, 35], is represented with a green curve.

- Cohesive fracture with depinning energy (brown curve) requiring a finite energy dissipation to nucleate the crack (depinning energy) and a further dissipation to increase the displacement jump (as in Barenblatt's model).

We show that it is possible to obtain the four macroscopic behaviours above within the same model by a proper tuning of the constitutive functions, that affect the order of the appearance of the elastic $(E)$, plastic $(P)$, damage $(D)$, or coupled $(P D)$ phases during the evolution. The cohesive behaviour is associated to the appearance of localised plastic deformations at the centre of damaged bands. For sake of simplicity, we will consider in the present work only a one-dimensional traction problem, that allows a throughout comparison between (semi-)analytical and numerical results. Many of the fundamental features of the model can be highlighted in this simplified setting.

The paper is structured as follows. In Sec. 2, the main ingredients of the gradient damage-plasticity model of [5, 6] are recalled. We deduce within a variational framework the evolution laws, where the displacement and the gradient of damage fields are allowed to be discontinuous in space. The homogeneous material response, obtained

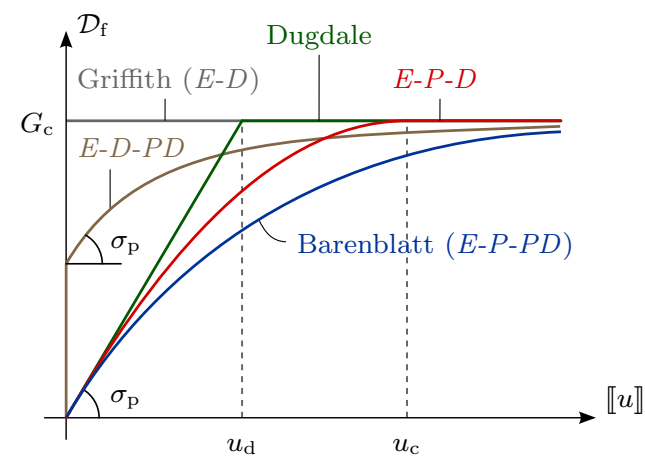

(a)

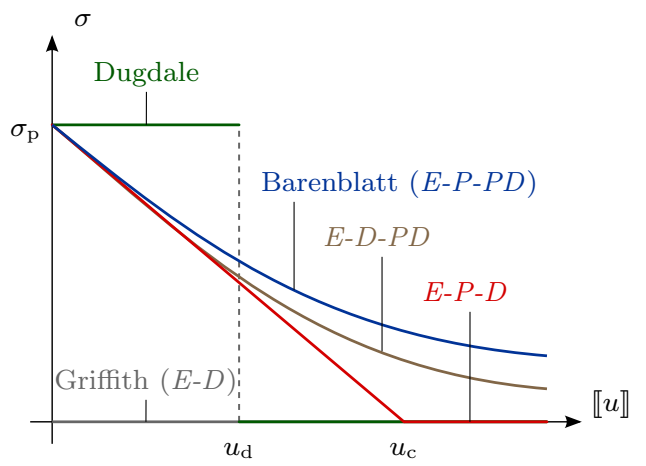

(b)

Figure 1: Dependence of the fracture energy (a) and the stress (b) on the crack opening $\llbracket u \rrbracket$ obtained with different choices of the constitutive functions. The macroscopic response ranges from purely brittle to ductile, including Barenblatt-like and Dugdale-like cohesive behaviours. Each behaviour corresponds to different sequences of evolution stages, namely: $E-D, E-P-D, E-P-P D$ and $E-D-P D$ (see Sec. 3 for the notation). $G_{\mathrm{c}}$ denotes the toughness, defined as the fracture energy dissipation for $\llbracket u \rrbracket \rightarrow \infty$.

by assuming that strains, plastic strains and damage are uniform in space, is discussed in Sec. 3. The aim is twofold: whilst being of fundamental importance to derive the localised responses, studying the homogeneous response also unveils the capability of the model to describe different sequences in the material evolution. Structural problems, showing localised responses, are tackled in Sec. 5, after describing the numerical implementation (Sec. 4). For a one-dimensional displacement-controlled traction test, we compare the analytical results and numerical simulations, and discuss the role of the internal length penalising the gradient damage term. We show that different constitutive choices of the constitutive parameters lead to the global fracture responses sketched in Fig. 1 .

Regarding the notation, the prime will denote either the derivative with respect to the spatial variable $x$ or the derivative with respect to the damage parameter, the dot stands for the time derivative; for instance $u^{\prime}(x, t)=$ $\partial u(x, t) / \partial x, \sigma_{\mathrm{P}}^{\prime}(\alpha)=\mathrm{d} \sigma_{\mathrm{P}}(\alpha) / \mathrm{d} \alpha$ and $\dot{u}(x, t)=\partial u(x, t) / \partial t$. 


\section{The formulation of a gradient damage model coupled with plasticity}

In this section, we briefly recall the ingredients and the main results of the gradient damage model coupled with plasticity presented in [5, 6]. The model is developed in a rate-independent framework. As such, viscous phenomena are not taken into account whilst plasticity and damage evolutions are assumed time-scale invariant. Moreover, inertial effects are neglected and the external actions are assumed smooth in time, so as to comply with a quasi-static setting.

\subsection{State variables and basic energetic quantities}

Let us consider, in an infinitesimal strain setting, a onedimensional straight bar whose reference configuration is the open bounded domain $(0, L)$. We assume that each point of the body $x \in(0, L)$ is characterized by the state variables described in Tab. 1. The bar is subjected to Dirichlet boundary conditions $u=0$ in $x=0$ and $u=$ $U(t)$ in $x=L$. We consider only monotonically increasing loadings with $t$ and, up to a re-parametrization of the time variable, we set $U(t)=t L$.

\begin{tabular}{lll}
\hline state variable & type \\
\hline$u$ & displacement & observable \\
$\varepsilon$ & total strain & observable, dependent \\
$p$ & plastic strain & internal, reversible \\
$\bar{p}$ & accumulated plastic & internal, irreversible, \\
& strain & dependent \\
$\alpha$ & damage & internal, irreversible \\
$\alpha^{\prime}$ & gradient of damage & internal, dependent \\
\hline
\end{tabular}

Table 1: State variables.

The time-dependent state variables are scalar fields representing the displacement $u(x, t)$, the plastic strain $p(x, t)$ and the damage $\alpha(x, t)$. The infinitesimal strain and the accumulated plastic strain are given by

$$
\varepsilon(x, t)=u^{\prime}(x, t) \quad \text { and } \quad \bar{p}(x, t)=\int_{0}^{t}|\dot{p}(x, \tau)| \mathrm{d} \tau .
$$

The scalar damage field is assumed to be bounded in $[0,1]$, with

$$
\begin{cases}\alpha=0, & \text { undamaged material } \\ \alpha=1, & \text { fully damaged material }\end{cases}
$$

and must satisfy the following irreversibility condition 27.

$$
\dot{\alpha} \geq 0, \quad \text { on }[0, L] .
$$

The internal potential energy density is assumed to be

$$
\psi(\varepsilon, p, \alpha)=\frac{1}{2} \mathrm{E}(\alpha)(\varepsilon-p)^{2},
$$

where the constitutive function $\mathrm{E}(\alpha)$ represents the Young modulus of the material and is supposed to be sufficiently smooth and decreasing with $\alpha$ up to zero [59]. Accordingly, the stress is defined as

$$
\sigma:=\partial_{\varepsilon} \psi=\mathrm{E}(\alpha)(\varepsilon-p) \text {. }
$$

In the framework of Generalized Standard Materials, [44, the dissipative behaviour is described through the dissipation potential, which is a convex and positive function of the internal state variables rates, vanishing for null rates and possibly depending on the internal variables itself. Rate-independence is ensured by assuming the dissipation potential being 1-homogeneous with respect to the rates [54]. For the present model, the dissipation potential is assumed as the the sum of two contributions, the first due only to the accumulated plastic strain rate while the second one depending only on the damage and gradient of damage rates, namely

$$
\varphi\left(\bar{p}, \alpha, \alpha^{\prime}, \dot{p}, \dot{\alpha}, \dot{\alpha}^{\prime}\right)=\varphi_{\mathrm{p}}(\alpha, \dot{p})+\varphi_{\mathrm{d}}\left(\bar{p}, \alpha, \alpha^{\prime}, \dot{\alpha}, \dot{\alpha}^{\prime}\right),
$$

with

$$
\begin{aligned}
& \varphi_{\mathrm{p}}(\alpha, \dot{p}):=\sigma_{\mathrm{P}}(\alpha)|\dot{p}|, \\
& \varphi_{\mathrm{d}}\left(\bar{p}, \alpha, \alpha^{\prime}, \dot{\alpha}, \dot{\alpha}^{\prime}\right):=\left(\mathrm{w}^{\prime}(\alpha)+\sigma_{\mathrm{P}}^{\prime}(\alpha) \bar{p}\right) \dot{\alpha}+2 \mathrm{w}_{1} \ell^{2} \alpha^{\prime} \dot{\alpha}^{\prime} .
\end{aligned}
$$

Therein, the scalar function $\sigma_{\mathrm{P}}(\alpha)$ can be identified with the plastic yield stress in a uniaxial traction test. We require $\sigma_{\mathrm{P}}(\alpha)$ to be decreasing with the damage and vanishing for a fully damaged material. Consequently, we assume

$$
\begin{array}{ll}
\sigma_{\mathrm{P}}(0)=\sigma_{\mathrm{p}}>0, & \sigma_{\mathrm{P}}^{\prime}(\alpha)<0, \\
\sigma_{\mathrm{P}}(1)=0, & \sigma_{\mathrm{P}}^{\prime}(1) \leq 0,
\end{array}
$$

with $\sigma_{\mathrm{p}}$ being the plastic yield stress of the undamaged material. The scalar damage constitutive function $\mathrm{w}(\alpha)$ can be interpreted as the density of the energy dissipated by , the material during a homogeneous damage process (such that $\alpha^{\prime}(x)=0$ ), where the damage variable of the material point grows from 0 to $\alpha ; \mathrm{w}_{1}=\mathrm{w}(1)$ represents the specific fracture energy [28]. Accordingly, we assume $\mathrm{w}(\alpha)$ to be sufficiently smooth and to verify the following requirements:

$$
\mathrm{w}(0)=0, \quad \mathrm{w}^{\prime}(\alpha)>0, \quad \mathrm{w}_{1}<+\infty .
$$

One can easily prove that with these hypotheses $\varphi$ is never negative [5].

The dependence of $\varphi_{\mathrm{p}}$ on $\alpha$ and of $\varphi_{\mathrm{d}}$ on $\bar{p}$ introduces a coupling between plasticity and damage. Here, the underlying plasticity model is perfect plasticity. Nevertheless, hardening effects can be easily added, as done in [66. The second term in the damage dissipation potential part can be recognized as the standard gradientdamage contribution to the brittle-fracture regularization model, [23], with $\ell>0$ having the role of an internal material length related to the width of a damage localization profile. 
The total dissipated work up to an instant $t$ is, in general, a process dependent function. Nevertheless, for the dissipation potential in (4) and (5), the dissipated work turns out to be a state function. By straightforward calculations, we get

$$
\begin{aligned}
& \mathcal{D}\left(\left\{p_{\tau}\right\}_{\tau<t},\left\{\alpha_{\tau}\right\}_{\tau<t}\right):= \\
& =\int_{0}^{\mathrm{L}}\left(\int_{0}^{t} \varphi\left(\bar{p}_{\tau}, \alpha_{\tau}, \alpha_{\tau}^{\prime}, \dot{p}_{\tau}, \dot{\alpha}_{\tau}, \dot{\alpha}_{\tau}^{\prime}\right) \mathrm{d} \tau\right) \mathrm{d} x \\
& =\int_{0}^{\mathrm{L}} \int_{0}^{t} \frac{\mathrm{d}}{\mathrm{d} \tau}\left(\sigma_{\mathrm{P}}\left(\alpha_{\tau}\right) \bar{p}_{\tau}+\mathrm{w}\left(\alpha_{\tau}\right)+\mathrm{w}_{1} \ell^{2}\left(\alpha_{\tau}^{\prime}\right)^{2}\right) \mathrm{d} \tau \mathrm{d} x \\
& =\int_{0}^{\mathrm{L}}\left(\sigma_{\mathrm{P}}\left(\alpha_{t}\right) \bar{p}_{t}+\mathrm{w}\left(\alpha_{t}\right)+\mathrm{w}_{1} \ell^{2}\left(\alpha_{t}^{\prime}\right)^{2}\right) \mathrm{d} x \\
& :=\mathcal{D}\left(\bar{p}_{t}, \alpha_{t}\right)
\end{aligned}
$$

where the dependence of the state variables on the spatial coordinate $x$ has been omitted for sake of conciseness and $p_{t}=p(x, t), \alpha_{t}=\alpha(x, t), \bar{p}_{t}=\bar{p}(x, t)$. Therefore, in this particular case, we can introduce a total internal energy density since the dissipated work can be expressed as a state function, namely

$$
\begin{aligned}
& W\left(\varepsilon, p, \bar{p}, \alpha, \alpha^{\prime}\right)= \\
& \quad=\frac{1}{2} \mathrm{E}(\alpha)(\varepsilon-p)^{2}+\sigma_{\mathrm{P}}(\alpha) \bar{p}+\mathrm{w}(\alpha)+\mathrm{w}_{1} \ell^{2}\left(\alpha^{\prime}\right)^{2},
\end{aligned}
$$

with the corresponding total internal energy

$$
\mathcal{W}(u, p, \bar{p}, \alpha)=\int_{0}^{L} W\left(u^{\prime}(x), p(x), \bar{p}(x), \alpha(x), \alpha^{\prime}(x)\right) \mathrm{d} x,
$$

coinciding with the functional considered in [6].

Remark 1 (Plastic, damage and fracture dissipation). Despite the dissipated work is a state function, the single contributions due to plasticity and damage are not. We define the plastic and damage dissipated work by

$$
\mathcal{D}_{\mathrm{p}}(t):=\mathcal{D}_{\mathrm{p}}\left(\left\{p_{\tau}\right\}_{\tau<t},\left\{\alpha_{\tau}\right\}_{\tau<t}\right)=\int_{0}^{L} \int_{0}^{t} \sigma_{\mathrm{P}}\left(\alpha_{\tau}\right)\left|\dot{p}_{\tau}\right| \mathrm{d} \tau \mathrm{d} x,
$$

and

$$
\begin{aligned}
& \mathcal{D}_{\mathrm{d}}(t):=\mathcal{D}_{\mathrm{d}}\left(\left\{p_{\tau}\right\}_{\tau<t},\left\{\alpha_{\tau}\right\}_{\tau<t}\right)= \\
& =\int_{0}^{L} \int_{0}^{t}\left(\left(\mathrm{w}^{\prime}\left(\alpha_{\tau}\right)+\sigma_{\mathrm{P}}^{\prime}\left(\alpha_{\tau}\right) \bar{p}_{\tau}\right) \dot{\alpha}_{\tau}+\mathrm{w}_{1} \ell^{2}\left(\alpha_{\tau}^{\prime}\right)^{2}\right) \mathrm{d} \tau \mathrm{d} x .
\end{aligned}
$$

In addition, we define the fracture energy as

$$
\begin{aligned}
& \mathcal{D}_{\mathrm{f}}(t):=\mathcal{D}_{\mathrm{f}}\left(\left\{p_{\tau}\right\}_{\tau<t},\left\{\alpha_{\tau}\right\}_{\tau<t}\right)= \\
& \mathcal{D}\left(\left\{p_{\tau}\right\}_{\tau<t},\left\{\alpha_{\tau}\right\}_{\tau<t}\right)-\mathcal{D}_{\mathrm{p}}\left(\left\{p_{\tau}^{\mathrm{R}}\right\}_{\tau<t},\left\{\alpha_{\tau}\right\}_{\tau<t}\right)
\end{aligned}
$$

where we use the decomposition of the plastic strain in regular $p^{\mathrm{R}}$ and singular parts, which will be introduced below. With such a choice, only the singular part of the plastic strain is accounted, as responsible of the cohesive behaviour, in the fracture energy.

\subsection{Function spaces and decomposition in regular and sin- gular parts}

The global state field $\boldsymbol{\xi}:=(u, p, \bar{p}, \alpha)$ should be sufficiently smooth in space to keep the total energy 10 finite. Specifically, the space of the admissible damage fields is

$$
\mathcal{A}:=\left\{\alpha \in \mathrm{H}^{1}([0, L], \mathbb{R}): 0 \leq \alpha \leq 1\right\} .
$$

The damage field must be continuous, while its derivative may suffer discontinuities. The latter fact will play a fundamental role in describing cohesive fracture responses. The elastic strain field $\left(u^{\prime}-p\right)$ must be square integrable, i.e. to belong to $\mathrm{L}^{2}([0, L])$. For perfect plasticity, $p$ can localise and, consequently, induce similar singularities on $u^{\prime}$, 64, 33, 37, 38. Specifically, admissible plastic strains are in a subspace of the finite Radon measures $\mathcal{M}_{b}$, 36, 45]:

$$
\mathcal{P}:=\left\{p \in \mathcal{M}_{b}([0, L], \mathbb{R})\right\} .
$$

Consequently the admissible displacement fields are Special functions of Bounded Variations (SBV):

$$
\mathcal{U}(t):=\{u \in \operatorname{SBV}([0, L], \mathbb{R}): u(L)=L t\} .
$$

Then, the global state field $\boldsymbol{\xi}$ is piecewise smooth and its singular part is localized on a $\boldsymbol{\xi}$-dependent set $J(\boldsymbol{\xi}) \in$ $[0, L]$, called the jumps set of $\boldsymbol{\xi}$, which contains a finite number of smooth and non-intersecting points in $[0, L]$. The domain $[0, L] \backslash J(\boldsymbol{\xi})$ will be denoted regular domain and, for a sake of simplicity, we also assume that this set has zero intersection with the boundary $\{0, L\}$.

The displacement field is continuously differentiable on $[0, L] \backslash J(\boldsymbol{\xi})$ and admits jump discontinuities of finite amplitude on $J(\boldsymbol{\xi})$. Consistently, the strain, the plastic strain and the accumulated plastic strain fields can be decomposed into their regular part denoted $(\cdot)^{\mathrm{R}}$ and their singular (or jump) part denoted $(\cdot)^{\mathrm{S}}$. Specifically, the total strain is decomposed as

$$
\varepsilon=\varepsilon^{\mathrm{R}}+\varepsilon^{\mathrm{S}}, \quad \text { with } \quad \varepsilon^{\mathrm{S}}=\llbracket u \rrbracket \delta_{J(\boldsymbol{\xi})},
$$

and $\varepsilon^{\mathrm{R}}$ being the absolute continuous part of the gradient of the displacement field and $\delta$ the Dirac measure concentrated on the jumps set $J(\boldsymbol{\xi})$. Similarly, the plastic strain and the accumulated plastic strain fields are decomposed as,

$$
p=p^{\mathrm{R}}+p^{\mathrm{S}} \quad \text { and } \quad \bar{p}=\bar{p}^{\mathrm{R}}+\bar{p}^{\mathrm{S}},
$$

with

$$
p^{\mathrm{S}}=\llbracket u \rrbracket \delta_{J(\boldsymbol{\xi})}, \quad \bar{p}^{\mathrm{S}}=\int_{0}^{t}|\llbracket \dot{u} \rrbracket| \delta_{J(\boldsymbol{\xi})} \mathrm{d} \tau=\bar{P} \delta_{J(\boldsymbol{\xi})},
$$


$\bar{P}$ being the accumulated plastic strain density.

\subsection{Evolution laws}

Following the energetic formulation of rate independent systems [55, we define the solutions of the evolution problem as the processes $\boldsymbol{\xi}_{t}$ satisfying the following three energetic principles:

- Irreversibility:

$$
\dot{\alpha} \geq 0, \quad \text { on }[0, L], \quad \forall t \in(0, T) \text {. }
$$

- Stability:

$$
\mathcal{W}^{\prime}(u, p, \bar{p}, \alpha)(\widetilde{u}, \widetilde{p},|\widetilde{p}|, \widetilde{\alpha}) \geq 0
$$

for any admissible test direction $\widetilde{\boldsymbol{\xi}}=(\widetilde{u}, \widetilde{p},|\widetilde{p}|, \widetilde{\alpha})$ with $\mathcal{W}^{\prime}(a)(b)$ denoting the directional derivative of $\mathcal{W}$ in the point $a$ in the direction $b$.

- Energy balance:

$$
\frac{\mathrm{d}}{\mathrm{d} t}(\mathcal{W}(u, p, \bar{p}, \alpha)-\mathcal{L}([0, t]))=0, \quad \forall t \in[0, T]
$$

where

$$
\mathcal{L}([0, t])=\int_{0}^{t} f_{r}(\tau) \dot{U}(\tau) \mathrm{d} \tau,
$$

is the external power of the reaction force $f_{r}$ in $x=$ $L$.

From this formulation we derive a necessary set of local evolution laws, which are summarized in Tab. 2. The yield function of the damage field in the regular domain includes a gradient term, while the yield function of the damage field in the singular domain establishes the relation between the displacement jump, through the singular part of the plastic strain field, and the jump of the derivative of the damage field. The explicit derivation of these laws can be found in [5, 6]. The reader is also referred to [58, 60, 3, 2, 7, 4, for further details.

\subsection{Specific models}

From now on, the presentation is limited to a simple one parameter class of models. Specifically, the constitutive functions are chosen as follows,

$\mathrm{E}(\alpha)=(1-\alpha)^{2} \mathrm{E}_{0}, \quad \mathrm{w}(\alpha)=\mathrm{w}_{1} \alpha, \quad \sigma_{\mathrm{P}}(\alpha)=(1-\alpha)^{s} \sigma_{\mathrm{p}}$

where $\mathrm{w}_{1}$ and $\sigma_{\mathrm{p}}$ are two positive constitutive constants and $\mathrm{E}_{0}$ the Young modulus of the sound material. Renormalisation arguments allow us to set $\mathrm{E}_{0}=1$ without loss of generality. The functions (21) are different from those considered in [5, where they were chosen to allow for explicit analytical results. The present model reduces to the ones commonly used in a phase-field regularisation of brittle fracture [57, 49, 25] when neglecting plasticity $\left(\sigma_{\mathrm{p}} \rightarrow \infty\right)$. The underlying damage model is that of a strongly brittle material 60, for which the energy necessary to break the material is finite, while the underlying plastic model is that of perfect plasticity.

The exponent $s>0$ will tune the softening behaviour on the plastic yield stress. Its values will greatly influence the material response, as shown in the following section. In the rest of this paper, we will study the response of the bar for the specific choices of $\left(s, \sigma_{\mathrm{p}}, \mathrm{w}_{1}\right)$ listed in Tab. 3. As will be shown in the next section, since $\mathrm{w}^{\prime}(0)>0$, see 60, all these choices will imply an initial purely elastic stage in the response, noted as $(E)$. After the initial elastic stage, when reaching the initial yield stress, several evolutions are possible. They will include phases where only plastic strains evolves $(P)$, only damage evolves $(D)$, or with a coupled evolution of plasticity and damage $(P D)$.

\section{Homogeneous material responses}

We present in this Section the homogeneous responses for the models in Tab. 3. They are obtained by assuming that the damage and the strain fields are uniform in space. Using the boundary conditions on the displacement, one can immediately deduce that $\varepsilon=t$. Hence, the only unknowns of the response are then the plastic strain and the damage state variables, since $p_{t}=\bar{p}_{t}$. In addition, the material is initially assumed unstretched, not plasticized and undamaged, namely

$$
\boldsymbol{\xi}_{0}=\left(\varepsilon_{0}, p_{0}, \bar{p}_{0}, \alpha_{0}\right)=(0,0,0,0) .
$$

All responses are calculated using the stress-strain relation $\sigma=\mathrm{E}(\alpha)(t-p)$, the damage and plastic KarushKuhn-Tucker (KKT) systems in Tab. 2 Specifically, the yield stresses for plasticity $\left(\sigma_{\mathrm{P}}\right)$ and damage $\left(\sigma_{\mathrm{D}}\right)$ can be deduced from the yield conditions and read as

$$
\begin{aligned}
\sigma_{\mathrm{P}}(\alpha) & =(1-\alpha)^{s} \sigma_{\mathrm{p}}, \\
\sigma_{\mathrm{D}}(\bar{p}, \alpha) & =\sqrt{\mathrm{E}_{0}\left(\mathrm{w}_{1}-s(1-\alpha)^{s-1} \sigma_{\mathrm{p}} \bar{p}\right)} \sqrt{(1-\alpha)^{3}} .
\end{aligned}
$$

Responses for which $\sigma_{\mathrm{P}}(0)>($ resp. $<) \sigma_{\mathrm{D}}(0,0)$ will be hereafter denoted $E-D_{-}{ }^{*}$ (resp. $E-P_{-}{ }^{*}$ ) since damage (resp. plasticity) is first triggered after the initial elastic stage. Tab. 4 resumes the numerical values for the yield stresses and strains for the four models in Tab. 3. Some models show a secondary yielding, where plasticity (or damage) is triggered during a damage (or plastic) phase, see below. In the following we will synthetically present the key results for the four models by commenting the plots of Fig. 2 . Therein, the evolutions of stress, damage and plasticity are higlighted, as well as yield stresses and possible unloading paths.

\subsection{E-D model}

Since $\sigma_{\mathrm{P}}(0)>\sigma_{\mathrm{D}}(0,0)$, after the initial elastic stage a damaging stage occurs. Moreover, for any damage level, 


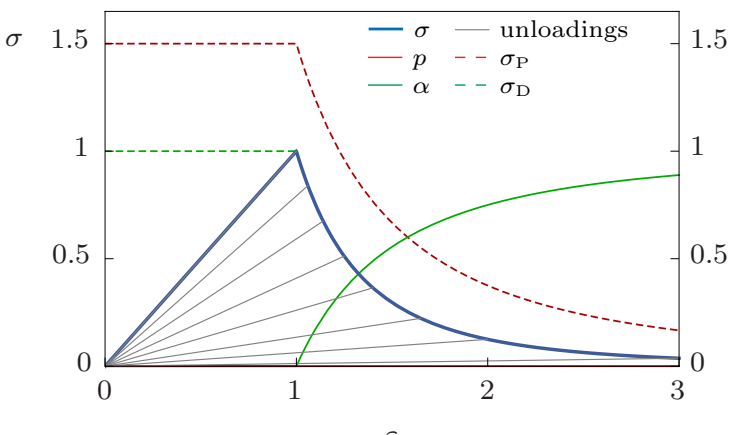

(a1) $\alpha, p$

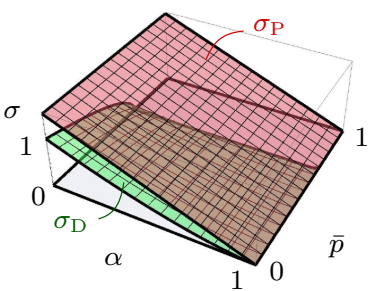

(a2)

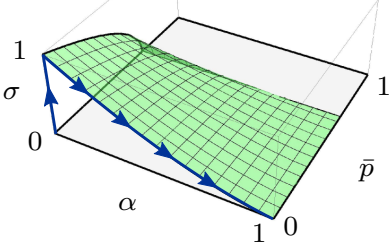

(a3)

(a) E-D model.

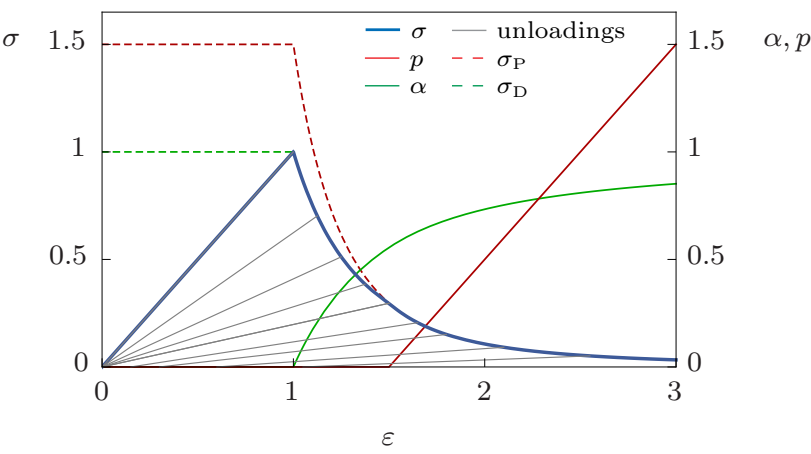

(b1)

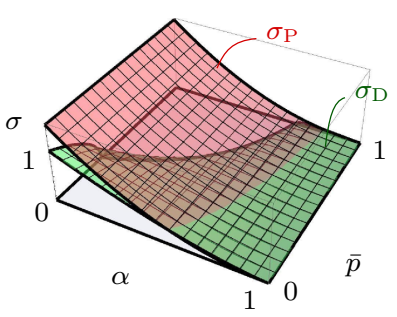

(b2)

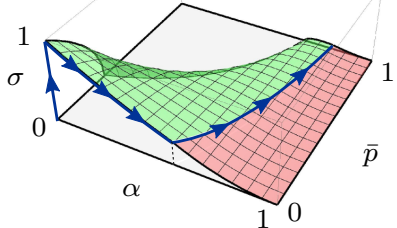

(b3)

(b) E-D-PD model.

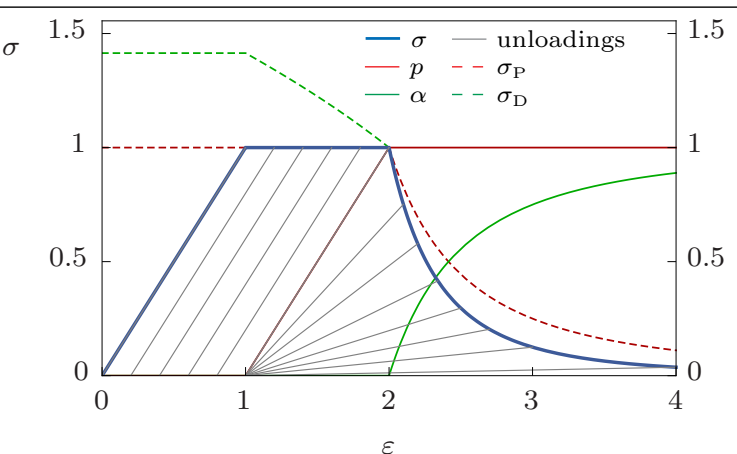

(c1)

\section{$\alpha, p$}

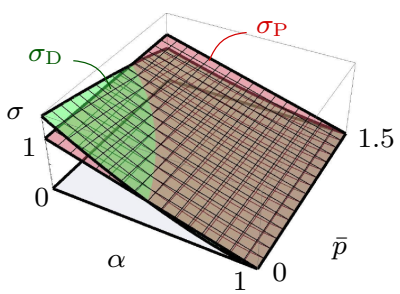

(c2)

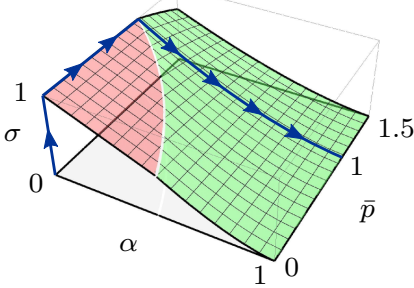

(c3)

(c) E-P-D model.

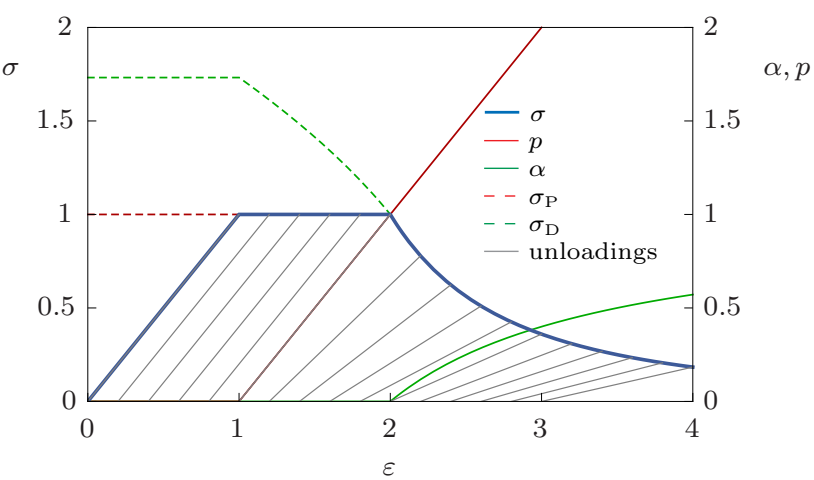

(d1)

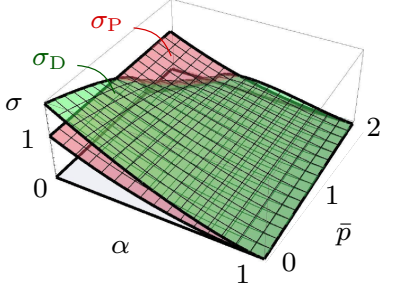

(d2)

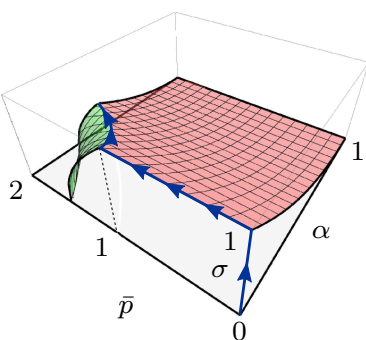

(d3)

(d) E-P-PD model.

Figure 2: Homogeneous responses for the constitutive choices of Tab. 3 For each model (rows) we show: the evolution of stress, plastic strain, damage and yield stresses with possible unloading curves; column 2, the plastic (red) and damage (green) yield surfaces; column 3, the actual plastic and damage evolution paths (-). 


\begin{tabular}{|c|c|}
\hline $\begin{array}{l}\text { equilibrium } \\
\text { equations }\end{array}$ & plasticity conditions \\
\hline \multicolumn{2}{|r|}{ regular domain $[0, L] \backslash J(\boldsymbol{\xi})$} \\
\hline \multirow[t]{6}{*}{$\sigma^{\prime}=0$} & KKT system: \\
\hline & $f_{\mathrm{p}}(\sigma, \alpha) \leq 0, \quad \dot{\bar{p}}^{\mathrm{R}} \geq 0, \quad f_{\mathrm{p}}(\sigma, \alpha) \dot{\bar{p}}^{\mathrm{R}}=0 \quad f_{\mathrm{d}}(\sigma, \bar{p}, \alpha) \leq 0, \quad \dot{\alpha} \geq 0, \quad f_{\mathrm{d}}(\sigma, \bar{p}, \alpha) \dot{\alpha}=0$ \\
\hline & yield function: \\
\hline & $f_{\mathrm{d}}(\sigma, \bar{p}, \alpha):=\frac{1}{2} \mathrm{~S}^{\prime}(\alpha) \sigma^{2}-\mathrm{w}^{\prime}(\alpha)-\sigma_{\mathrm{P}}^{\prime}(\alpha) \bar{p}^{\mathrm{R}}+2 \mathrm{w}_{1} \ell^{2} \alpha^{\prime \prime}$ \\
\hline & flow rule: \\
\hline & $\dot{p}=\dot{\bar{p}} \operatorname{sign}(\sigma)$ \\
\hline \multicolumn{2}{|r|}{ agular domain $J(\xi)$} \\
\hline \multirow[t]{7}{*}{$\llbracket \sigma \rrbracket=0$} & KKT system: \\
\hline & $f_{\mathrm{p}}^{\mathrm{S}}(\sigma, \alpha) \leq 0, \quad \dot{\bar{p}}^{\mathrm{S}} \geq 0, \quad f_{\mathrm{p}}^{\mathrm{S}}(\sigma, \alpha) \dot{\bar{p}}^{\mathrm{S}}=0 \quad f_{\mathrm{d}}^{\mathrm{S}}(\bar{p}, \alpha) \leq 0, \quad \dot{\alpha} \geq 0, \quad f_{\mathrm{d}}^{\mathrm{S}}(\bar{p}, \alpha) \dot{\alpha}=0$ \\
\hline & yield function: \\
\hline & $f_{\mathrm{d}}^{\mathrm{S}}(\bar{p}, \alpha):=2 \mathrm{w}_{1} \ell^{2} \llbracket \alpha^{\prime} \rrbracket-\sigma_{\mathrm{P}}^{\prime}(\alpha) \bar{P}$ \\
\hline & flow rule: \\
\hline & $\dot{p}^{\mathrm{S}}=\dot{\bar{p}}^{\mathrm{S}} \operatorname{sign}(\sigma)$ \\
\hline & boundary conditions $\{0, L\}$ \\
\hline$\left.\sigma\right|_{0}=f_{r}$ or $\left.u\right|_{0}=0$ & $\left.\alpha^{\prime}\right|_{0} \leq 0$ or $\left.\alpha\right|_{0}=0$ \\
\hline$\left.\sigma\right|_{L}=f_{r}$ or $\left.u\right|_{L}=t$ & $\left.\alpha^{\prime}\right|_{L} \geq 0$ or $\left.\alpha\right|_{L}=0$ \\
\hline
\end{tabular}

Table 2: Summary of the governing equations deduced from the energy balance eb), stability condition (st) and irreversibility condition (ir). In $f_{\mathrm{d}}(\sigma, \bar{p}, \alpha), \mathrm{S}(\alpha)=\mathrm{E}^{-1}(\alpha)$ represents the compliance elastic tensor.

\begin{tabular}{ccccccc}
\hline model name & $\sigma_{\mathrm{P}}(0)$ & $\sigma_{\mathrm{D}}(0,0)$ & $\varepsilon_{\mathrm{y}}$ & $\sigma_{\mathrm{y}}$ & $\varepsilon_{\mathrm{yy}}$ & $\sigma_{\mathrm{yy}}$ \\
\hline$E-D$ & $3 / 2$ & 1 & 1 & 1 & $/$ & $/$ \\
\hline$E-D-P D$ & $3 / 2$ & 1 & 1 & 1 & $3 / 2$ & $(2 / 3)^{3}$ \\
\hline$E-P-D$ & 1 & $\sqrt{2}$ & 1 & 1 & 2 & 1 \\
\hline$E-P-P D$ & 1 & $\sqrt{3}$ & 1 & 1 & 2 & 1 \\
\hline
\end{tabular}

Table 4: First $(\cdot)_{\mathrm{y}}$ and second $(\cdot)_{\text {yy }}$ yield points. Cells with a gray background highlight the smaller initial yield stress, $\sigma_{\mathrm{y}}$, that defines which inelastic phenomenon is triggered first.

one has $\sigma_{\mathrm{P}}(\alpha)>\sigma_{\mathrm{D}}(0, \alpha)$ and hence a second yield instant $\sigma_{\text {yy }}$ does not exist. The complete $E-D$ material response is described in Fig. 2a.

Fig. 2a1 shows that the damage and plastic yield stresses do not intersect. This means that the plastic yield stress is never reached and, therefore, plastic strains never evolve.

\section{2. $E-D-P D$ model}

Since $\sigma_{\mathrm{P}}(0)>\sigma_{\mathrm{D}}(0,0)$, after the initial elastic stage, a damaging stage occurs. Differently from the $E-D$ model, at a certain damage level, a second yield instant exists. Indeed, by imposing $\sigma_{\mathrm{P}}(\alpha)=\sigma_{\mathrm{D}}(0, \alpha)$, we get $\left(\varepsilon_{\mathrm{yy}}, \sigma_{\mathrm{yy}}\right)=$ $\left(\sigma_{\mathrm{p}} / \mathrm{E}_{0}, \mathrm{w}_{1}^{2} \mathrm{E}_{0}^{2} / \sigma_{\mathrm{p}}^{3}\right)$ and from that instant on, damage and plasticity must evolve together. The complete $E-D-P D$ material response is described in Tab. 5 and Fig. $2 \mathrm{~b}$.

\begin{tabular}{cccc}
\hline & $\mathrm{E}$ & $\mathrm{D}$ & $\mathrm{PD}$ \\
\hline$t$ & {$\left[0, \sqrt{\frac{\mathrm{w}_{1}}{\mathrm{E}_{0}}}\right]$} & {$\left[\sqrt{\frac{\mathrm{w}_{1}}{\mathrm{E}_{0}}}, \frac{\sigma_{\mathrm{p}}}{\mathrm{E}_{0}}\right]$} & {$\left[\frac{\sigma_{\mathrm{p}}}{\mathrm{E}_{0}},+\infty\right]$} \\
\hline$\sigma(t)$ & $\mathrm{E}_{0} t$ & $\frac{\mathrm{w}_{1}^{2}}{\mathrm{E}_{0} t^{3}}$ & $\frac{\mathrm{w}_{1}^{2} \mathrm{E}_{0}}{\sigma_{\mathrm{p}}\left(2 \mathrm{E}_{0} t-\sigma_{\mathrm{p}}\right)^{2}}$ \\
$p(t)$ & 0 & 0 & $t-\frac{\sigma_{\mathrm{p}}}{\mathrm{E}_{0}}$ \\
$\alpha(t)$ & 0 & $1-\frac{\mathrm{w}_{1}}{\mathrm{E}_{0} t^{2}}$ & $1-\frac{\mathrm{w}_{1} \mathrm{E}_{0}}{\sigma_{\mathrm{p}}\left(2 \mathrm{E}_{0} t-\sigma_{\mathrm{p}}\right)}$ \\
\hline
\end{tabular}

Table 5: Homogeneous response of the $E-D-P D$ model: evolution of the state variables with the loading in the elastic $(E)$, damage $(D)$, and coupled $(P D)$ phases. The $E-D$ is the particular case where $\sigma_{\mathrm{p}} \rightarrow \infty$ and $P D$ phase does not exists.

From the stress-strain response of Fig. 2b1, one can appreciate the existence of a second yield point where the yield stresses intersect. While during the damaging stage only a decrease of the stiffness occurs, after such second yield instant also the plastic strain starts to evolve together with damage, as highlighted by the increasing residual strains and contemporary decreasing stiffness of the 


\begin{tabular}{ccccl}
\hline model name & $\sigma_{\mathrm{p}}$ & $\mathrm{w}_{1}$ & $s$ & mechanical response \\
\hline$E-D$ & $3 / 2$ & 1 & 1 & Griffith's brittle fracturf \\
\hline$E-D-P D$ & $3 / 2$ & 1 & 2 & mixed Griffith's brittle/Barenblatt's cohesive fracture \\
\hline$E-P-D$ & 1 & 2 & 1 & plasticity with Dugdale's like cohesive fracture (ductile fracture) \\
\hline$E-P-P D$ & 1 & 3 & 2 & plasticity with Barenblatt's cohesive fracture (ductile fracture) \\
\hline
\end{tabular}

Table 3: The four choices of the constitutive parameters in 21) considered throughout this work and the associated qualitative responses.

unloading curves.

\section{3. $E-P-D$ model}

Since $\sigma_{\mathrm{P}}(0)<\sigma_{\mathrm{D}}(0,0)$, after the initial elastic stage, a plastic stage occurs. Since $\partial_{\bar{p}} \sigma_{\mathrm{D}}(\bar{p}, 0)<0$, due to the assumption (6), a second yield instant $\sigma_{\mathrm{yy}}$, where damage is triggered, always exists. By imposing $\sigma_{\mathrm{P}}(0)=\sigma_{\mathrm{D}}(\bar{p}, 0)$, we get $\left(\varepsilon_{\mathrm{yy}}, \sigma_{\mathrm{yy}}\right)=\left(\mathrm{w}_{1} / \sigma_{\mathrm{p}}, \sigma_{\mathrm{p}}\right)$. From that instant on, only damage evolves since the plastic yield stress decreases more slowly with $\alpha$ than the damage yield stress, $\partial_{\alpha} \sigma_{\mathrm{D}}\left(\bar{p}\left(\varepsilon_{\mathrm{yy}}\right), \alpha\right)<$ $\partial_{\alpha} \sigma_{\mathrm{P}}(\alpha)$. The complete $E-P-D$ material response is described in Tab. 6 and Fig. 2c.

\begin{tabular}{cccc}
\hline & $\mathbf{E}$ & $\mathrm{P}$ & $\mathrm{D}$ \\
\hline$t$ & {$\left[0, \frac{\sigma_{\mathrm{p}}}{\mathrm{E}_{0}}\right]$} & {$\left[\frac{\sigma_{\mathrm{p}}}{\mathrm{E}_{0}}, \frac{\mathrm{w}_{1}}{\sigma_{\mathrm{p}}}\right]$} & {$\left[\frac{\mathrm{w}_{1}}{\sigma_{\mathrm{p}}},+\infty\right]$} \\
\hline$\sigma(t)$ & $\mathrm{E}_{0} t$ & $\sigma_{\mathrm{p}}$ & $\frac{\sigma_{\mathrm{p}}^{4}}{\mathrm{E}_{0}^{3}(t-\bar{p})^{3}}$ \\
$p(t)$ & 0 & $t-\frac{\sigma_{\mathrm{p}}}{\mathrm{E}_{0}}$ & $\frac{\mathrm{w}_{1}}{\sigma_{\mathrm{p}}}-\frac{\sigma_{\mathrm{p}}}{\mathrm{E}_{0}}$ \\
$\alpha(t)$ & 0 & 0 & $1-\frac{\sigma_{\mathrm{p}}^{2}}{\mathrm{E}_{0}^{2}(t-\bar{p})}$ \\
\hline
\end{tabular}

Table 6: Homogeneous response of the $E-P-D$ model.

In contrast with the $E$ - $D$ case, Fig. 2c1 shows the existence of an intersection point between the damage and plastic thresholds represented by the dashed green and red curves, respectively. This point is associated to a second yielding where plasticity stops and damage starts to evolve. Indeed the unloading paths show first an increase of residual strains and then, only a decrease of the elastic stiffness.

\subsection{E-P-PD model}

Also for this case, since $\sigma_{\mathrm{P}}(0)<\sigma_{\mathrm{D}}(0,0)$, after the initial elastic stage not only a plastic stage occurs but also a second yield instant, coinciding with the one of the $E$ $P-D$ model, $\left(\varepsilon_{\mathrm{yy}}, \sigma_{\mathrm{yy}}\right)=\left(\mathrm{w}_{1} / \sigma_{\mathrm{p}}, \sigma_{\mathrm{p}}\right)$. But now, since the plastic yield stress decreases faster than the damage yield stress with respect to the damage level, $\partial_{\alpha} \sigma_{\mathrm{D}}\left(\bar{p}\left(\varepsilon_{\mathrm{yy}}\right), \alpha\right)>$ $\partial_{\alpha} \sigma_{\mathrm{P}}(\alpha)$, plasticity must evolve together with damage after that second yield instant. The general evolution, which equals the evolution of the $E-P-D$ response during the elastic-plastic stage, is described in Tab. 7 and Fig. 2d.

\begin{tabular}{cccc}
\hline & $\mathrm{E}$ & $\mathrm{P}$ & $\mathrm{PD}$ \\
\hline$t$ & {$\left[0, \frac{\sigma_{\mathrm{p}}}{\mathrm{E}_{0}}\right]$} & {$\left[\frac{\sigma_{\mathrm{p}}}{\mathrm{E}_{0}}, \frac{\mathrm{w}_{1}}{\sigma_{\mathrm{p}}}\right]$} & {$\left[\frac{\mathrm{w}_{1}}{\sigma_{\mathrm{p}}},+\infty\right]$} \\
\hline$\sigma(t)$ & $\mathrm{E}_{0} t$ & $\sigma_{\mathrm{p}}$ & $\frac{\mathrm{w}_{1}^{2} \mathrm{E}_{0}^{2}}{\sigma_{\mathrm{p}}\left(2 \mathrm{E}_{0} t-\sigma_{\mathrm{p}}\right)^{2}}$ \\
$p(t)$ & 0 & $t-\frac{\sigma_{\mathrm{p}}}{\mathrm{E}_{0}}$ & $t-\frac{\sigma_{\mathrm{p}}}{\mathrm{E}_{0}}$ \\
$\alpha(t)$ & 0 & 0 & $1-\frac{\mathrm{w}_{1} \mathrm{E}_{0}}{\sigma_{\mathrm{p}}\left(2 \mathrm{E}_{0} t-\sigma_{\mathrm{p}}\right)}$ \\
\hline
\end{tabular}

Table 7: Homogeneous response of the $E-P-P D$ model.

From the stress-strain response of Fig. 2d1, one can appreciate the existence of a second yield point where the yield stresses intersect. While during the plastic stage no damage occurs, after such second yield instant both plasticity and damage evolve. Indeed, the unloading paths show first an increasing of residual strains and then a coupled behaviour, with plastic strains that continue to increase with a progressive decrease of the elastic stiffness.

\section{Numerical solution algorithm}

To solve numerically the evolution problem we adopt a staggered algorithm suggested by the time-discrete version of the variational formulation. This can be considered as an extension of the consolidated alternate minimization scheme used in the regularised models of brittle fracture, [23, 22, 24, 13, 25, and fits the incremental energy minimization framework [56, 61]. Similar algorithms have been used for coupled plasticity-damage problems also by [10, 52, 62. Both the time and space discretisation procedures for the state variables fields are standard.

Time-discretisation. The time interval $[0, T]$ is discretised with possibly non-uniform time steps $\mathrm{d} t_{i}$. By definition, (1), the discretised version of the accumulated plastic strain at time $t$ becomes

$$
\bar{p}_{i}=\bar{p}_{i-1}+\left|p_{i}-p_{i-1}\right| .
$$

Hence, we find the fields $\left(u_{i}, p_{i}, \alpha_{i}\right)$ by solving the following minimisation problem at time step $t_{i}$

$$
\min _{u, p, \alpha}\left\{\mathcal{W}_{i}(u, p, \alpha), \alpha \geq \alpha_{i-1}, u(L)=t_{i}\right\}
$$


where

$$
\begin{aligned}
\mathcal{W}_{i}(u, p, \alpha)= & \int_{0}^{L}\left(\frac{1}{2} \mathrm{E}(\alpha)(\varepsilon-p)^{2}+\mathrm{w}(\alpha)+\mathrm{w}_{1} \ell^{2}\left(\alpha^{\prime}\right)^{2}\right) \mathrm{d} x \\
& +\int_{0}^{L} \sigma_{\mathrm{P}}(\alpha)\left(\left|p-p_{i-1}\right|+\bar{p}_{i-1}\right) \mathrm{d} x
\end{aligned}
$$

Space-discretisation. The displacement and damage fields are projected over a piecewise affine finite element space ( $P_{1}$ triangular elements) over the same unstructured mesh. Arguments for not using higher degree finite elements can be found, for example, in [21]. Conversely, the plastic strain field $p$ is projected over a discrete discontinuous space (quadrature elements ${ }^{1}$ ) due to the local character of the plasticity minimization problem. To get a better approximation of the optimal damage profile, the mesh size $h$ must be smaller than the internal length $\ell$ (at least $h \leq \ell / 4$ ), see [24, rapidly leading to the need of large scale computations in $2 \mathrm{D}$ and $3 \mathrm{D}$ settings.

Although (27) is non convex, it is convex with respect to each variable individually. Therefore, we alternate minimizations with respect to $u, p$ and $\alpha$, as described in Algorithms 1-2. In particular, we first optimize with respect to the displacements and plastic strains and then, only after convergence, with respect to the damage field. Specifically, the minimization step with respect to $u$ is equivalent to solve a linear elasticity problem. The minimization step with respect to $p$ is equivalent to a linear local projection, refer to Algo. 2, because of the perfect-plasticity setting. Instead, the minimization step with respect to $\alpha \in\left[\alpha_{i-1}, 1\right]$ requires a box-constrained minimization algorithm. The code has been written using the FEniCS library [50, 9] for finite elements and PETSc [16, 14, 15] for linear algebra and bound-constrained solvers. Of course, as the total energy is non convex, one cannot expect convergence of Algo. 1 to a global minimizer. However, one can prove that the alternate minimization process is unconditionally stable and globally decreasing and that it leads to a stationary point of 27) which may be a local (or global) minimizer or a saddle point for the energy. From a practical standpoint, we observe that the algorithm is robust with respect to the mesh discretisation, provided that the mesh size is small enough compared to the internal length.

The present model does not introduce any localization limiter for the plastic strain. However, as in perfect plasticity, the numerical results are convergent with respect to the mesh size. The plastic strain localises in a single mesh element. But, when decreasing the mesh-size, its absolute value increases so as to preserve a constant element-wise integral, which represents the displacement jump.

\footnotetext{
${ }^{1}$ In quadrature elements, the function values are defined only on Gauss integration points.
}

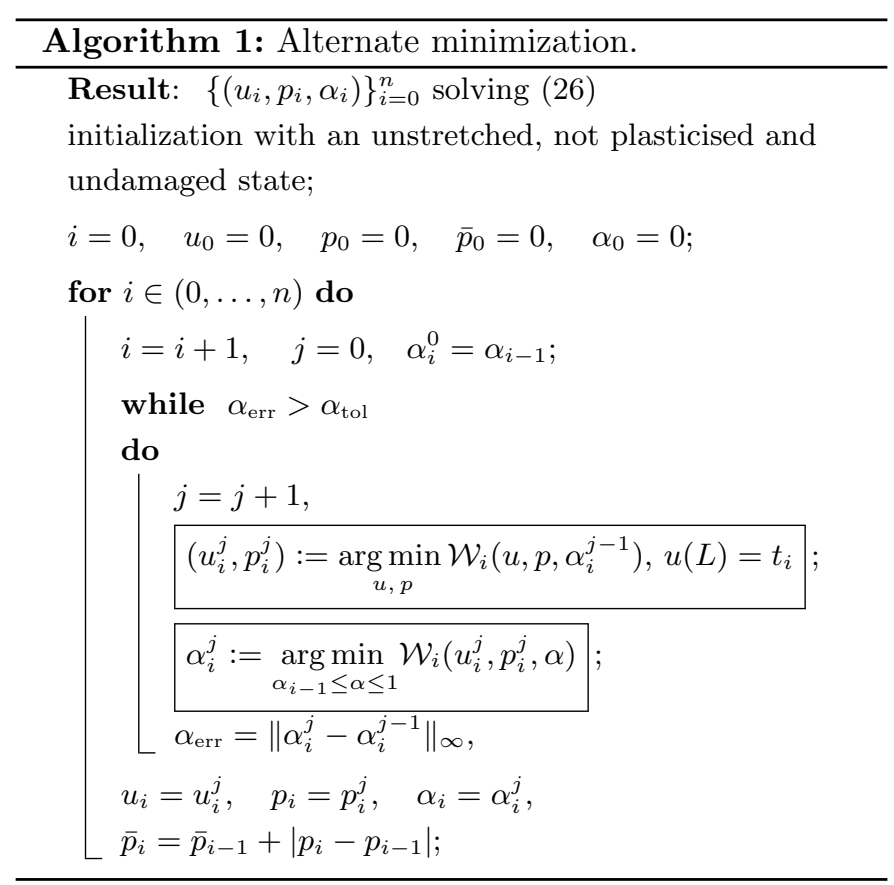

\section{Numerical simulations of the one-dimensional traction test}

This section presents the numerical solutions of the traction test. The bar, of unitary length $L=1$, is initially unstreched, not plasticized and undamaged. Its left-end is fixed while on its right-end, a monotonically increasing displacement $u(1)=t$ is prescribed, see Fig. 3. The gra-

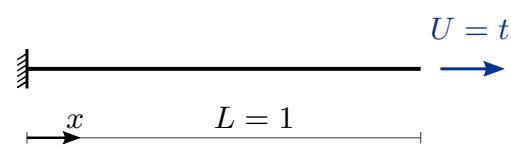

Figure 3: One-dimensional displacement-controlled traction bar test setup.

dient term plays a crucial role in the structural response, acting as a localisation limiter for the damage field. The value of the internal length, $\ell=0.15$, is chosen to be sufficiently small to allow for the development of a full localisation inside the bar. We use a uniform mesh with element size $h=1 / 200$. We perform numerical simulations for the parameters listed in Tab. 8, which are renormalised in order to give a common elastic limit at $t_{\mathrm{y}}=1, \sigma_{\mathrm{y}}=1$ for the four models.

We provide a throughout verification of the numerical solutions obtained with the algorithms of Sec. 4 against semi-analytical results. A discussion of the semi-analytical solution for the localisation problem is reported in Appendix A, see also [1].

\subsection{E-D response}

The $E-D$ model describes the occurrence of a brittle fracture without the occurrence of plastic strains. Its prop- 


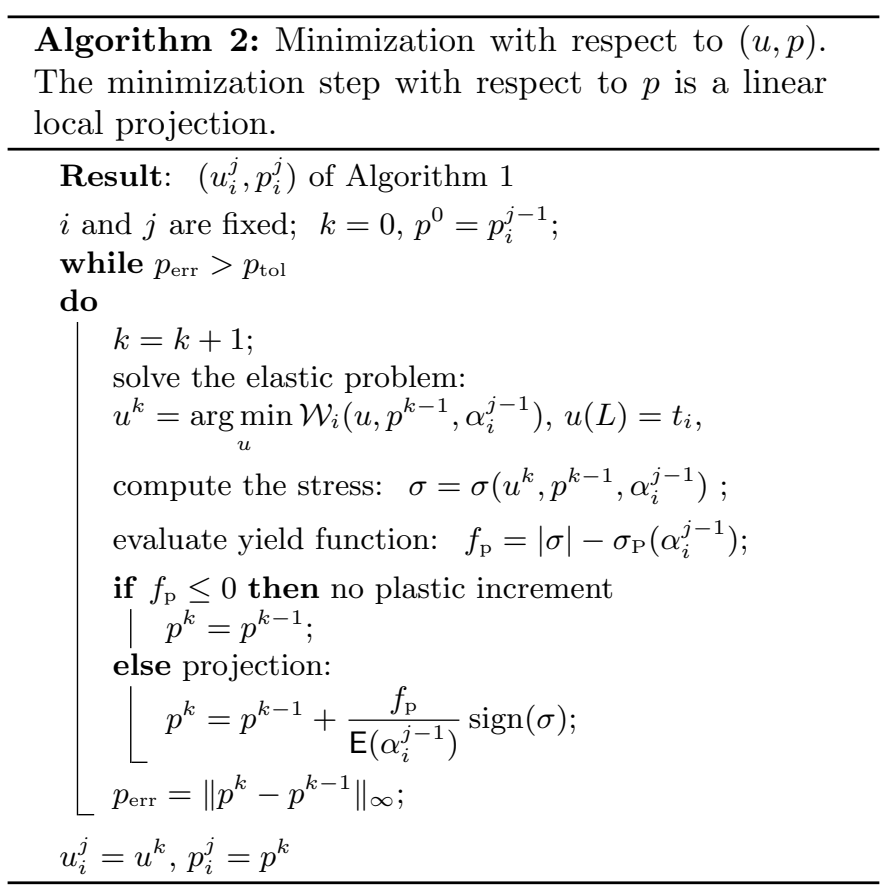

\begin{tabular}{ccccc}
\hline model name & $\sigma_{\mathrm{p}}$ & $\mathrm{w}_{1}$ & $s$ & $\ell / L$ \\
\hline$E-D$ & $>1$ & 1 & 1 & 0.15 \\
\hline$E-D-P D$ & 2 & 1 & 2 & 0.15 \\
\hline$E-P-D$ & 1 & 2 & 1 & $0.15 / 0.08$ \\
\hline$E-P-P D$ & 1 & 3 & 2 & 0.15 \\
\hline
\end{tabular}

Table 8: Constitutive parameters for the test cases of Sec. 5

erties have been widely discussed in previous works, when considering damage-gradient model as phase-field approximation of brittle fracture [57, 51. Here we recall and discuss some important facts, useful for later discussions. Fig. 4a displays the force-displacement response. In the numerical model, after the initial elastic stage, damage is triggered and grows instantaneously to its maximum level corresponding to a fractured state where all the dissipated energy is spent instantaneously. The analytical calculation uses the decreasing stress as parameter during the damaging stage. This allows the computation of the unstable snap-back branch in the force-displacement response. To follow this branch numerically would require specific pathfollowing techniques, 8. The energy plot of Fig. 4b shows a jump in the total energy at $t=1$, when the damage localisation appears. In the numerical simulation, the total energy is not conserved (but is decreasing). This inconsistency with the energy balance principle in the quasi-static rate-independent evolution is related to the use, in the time-discrete setting, a local stability criterion. It can be resolved by a more careful analysis, as discussed in [2].

The damage profile evolution is shown in Fig. 4c. The semi-analytical profiles have been determined through the technique resumed in Appendix A. The numerical solu- tion jumps from an undamaged state to the fully fractured stated with $\alpha=1$ at the center. Figure $4 \mathrm{c}$ shows that fully localised profiles of the analytical and numerical solutions are slightly different. In the numerical solution, the fully localised profile appears abruptly and accounts for the irreversibility condition only with respect to the previous time-step, i.e. $\alpha \geq 0$. Instead, the analytic calculation include the irreversibility conditions during the (unstable) growth of the damage profile, see Appendix A.1. However, the difference between the two profiles is barely noticeable and the dissipated energy in the two cases is almost indistinguishable. One can safely neglect this difference when performing more complex computations. This aspect will be further discussed in the $E-D-P D$ response. The jump of the displacement field in Fig. $4 \mathrm{~d}$ is the signature of the occurrence of a "crack" at the center of the localisation zone. All the solutions obtained translating the whole localization profile within the bar are energetically equivalent. The problem is obviously imperfection-sensitive and small perturbations of the material properties could alter the placement of the localization zone. However, the global structural response would not be affected by the a different position of the localization.

\section{2. $E-D-P D$ response}

In the $E-D-P D$ case, plasticity is triggered during the evolution of damage, leading to a coupled response, as seen for the corresponding homogeneous response. In the context of a structural problem, the fields can localise in space, leading to a subtler coupling. Specifically, a fracture occurs as soon as the damage-yield loading is reached, but the damage field does not reach the value 1 . The strain localisation is not due to the loss of stiffness, but to a singularity in the plastic strain (Dirac measure), that occurs at the center of the damage profile.

The force-displacement plot of Fig. 5 a shows that the stress never vanishes after the snap-back. Numerically, the progressive growth of the damage field, described in the analytical solution, is not observed. The analytical profiles have been built accounting for the irreversibility condition during their unstable growth, see Fig. A.15.

The energy plot in Fig. 5b discloses the key features of the coupled plasticity-damage evolution, that are more evident in Fig. 5c where the evolutions of the different energy contributions are plotted against the displacement jump due to the plastic localisation. Specifically, one can appreciate that some energy is dissipated by damage as soon as the discontinuity occurs while the remaining energy is progressively dissipated with the increase of the fracture opening. The plot of the stress against the displacement jump could be interpreted as a mixed Griffith's brittle/Barenblatt's cohesive fracture type: one needs a finite "depinning" energy to nucleate the crack, as in Griffith's model, and a further progressive dissipation to increase the crack opening. The displacements profiles in Fig. $5 \mathrm{f}$ gives a clear signature of the presence of a fracture. Again 


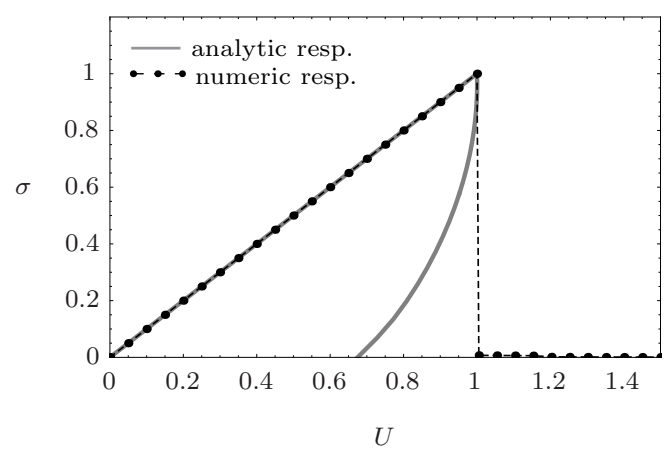

(a)

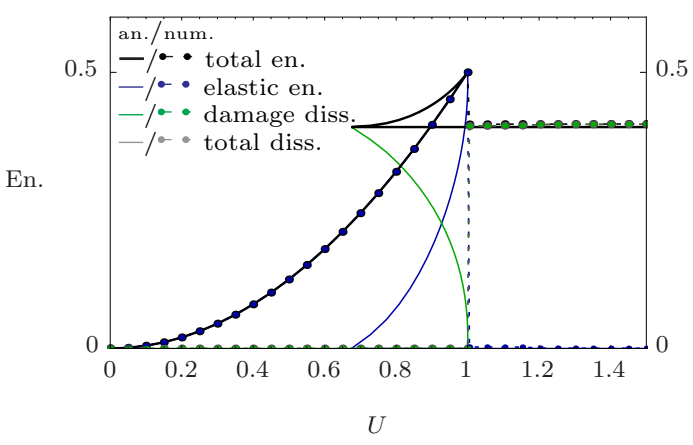

(b)

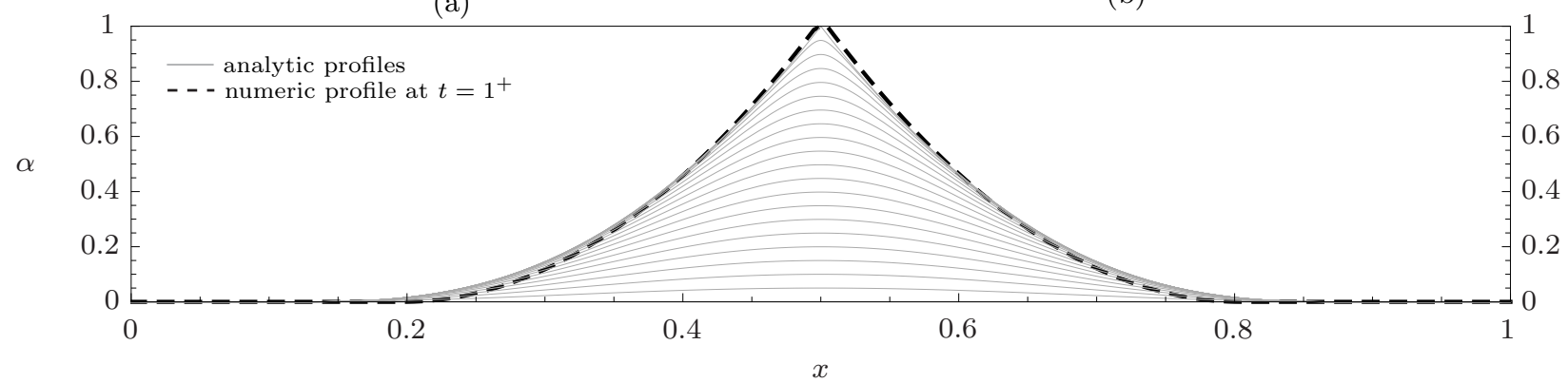

(c)

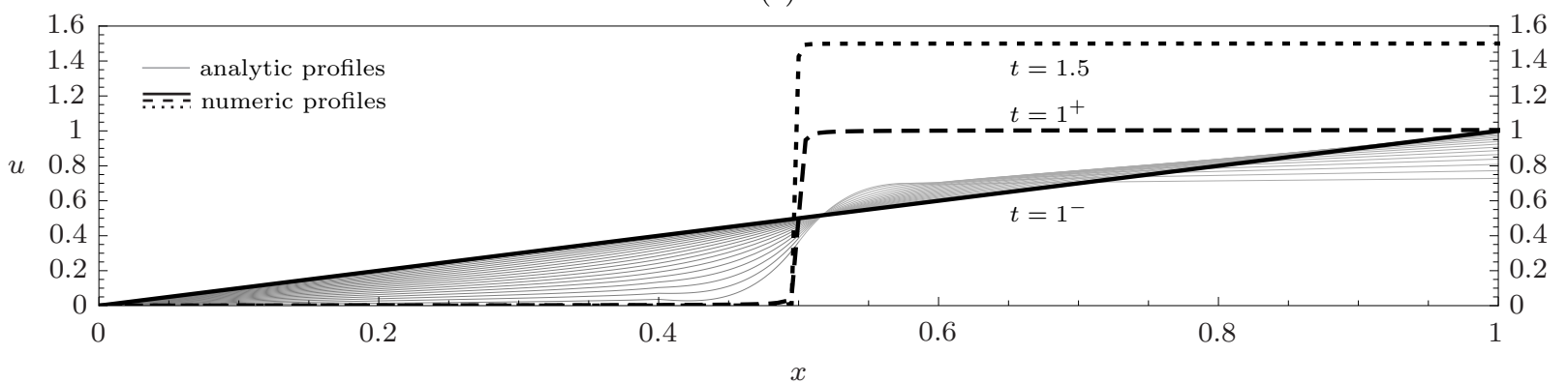

(d)

Figure 4: Numerical and analytical results for the traction test of the $E-D$ model: (a) force-displacement diagram; (b) energy diagrams; damage (c) and displacement (d) fields at different loading steps. 


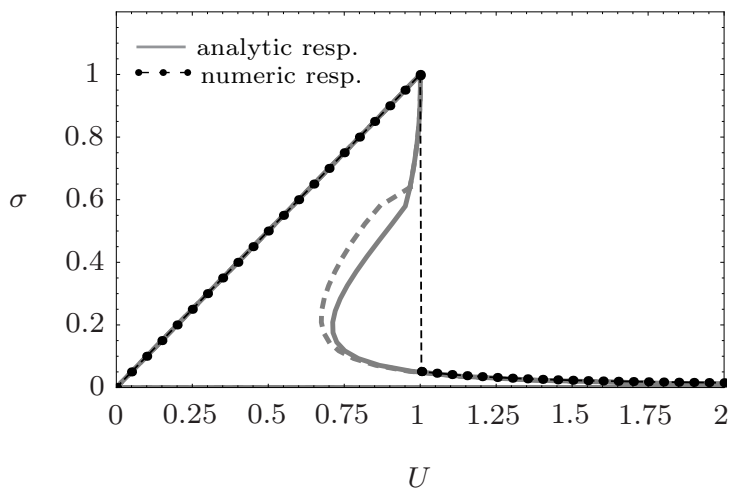

(a)

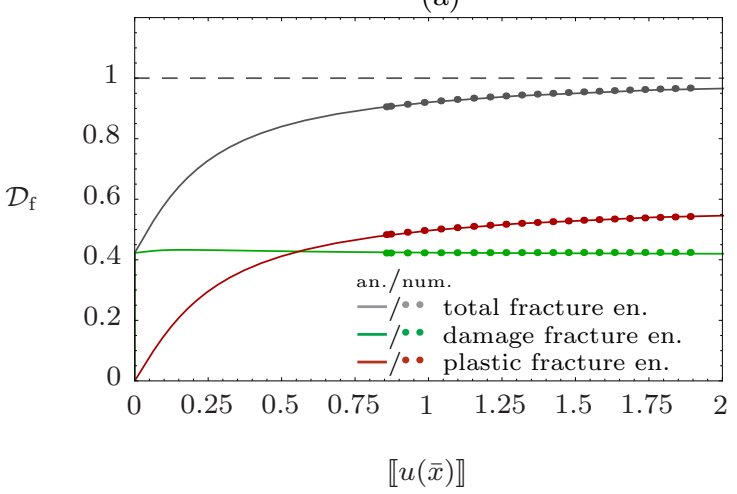

(c)

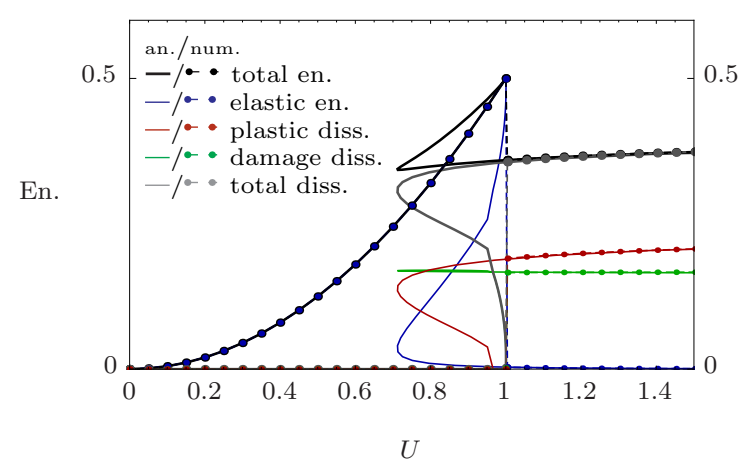

(b)

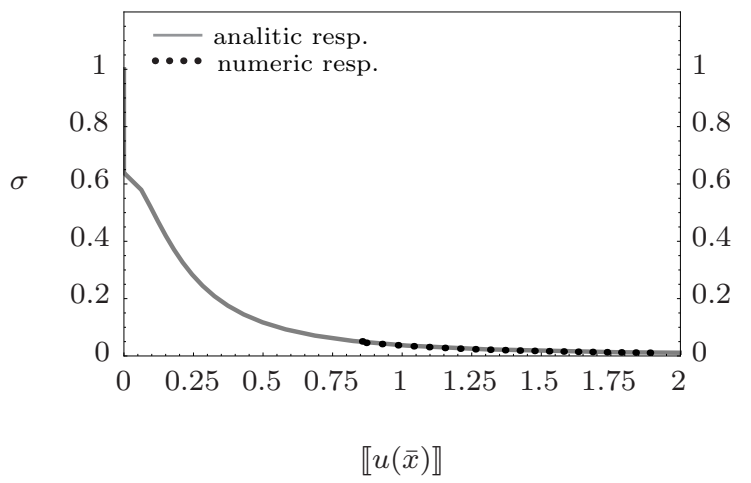

(d)

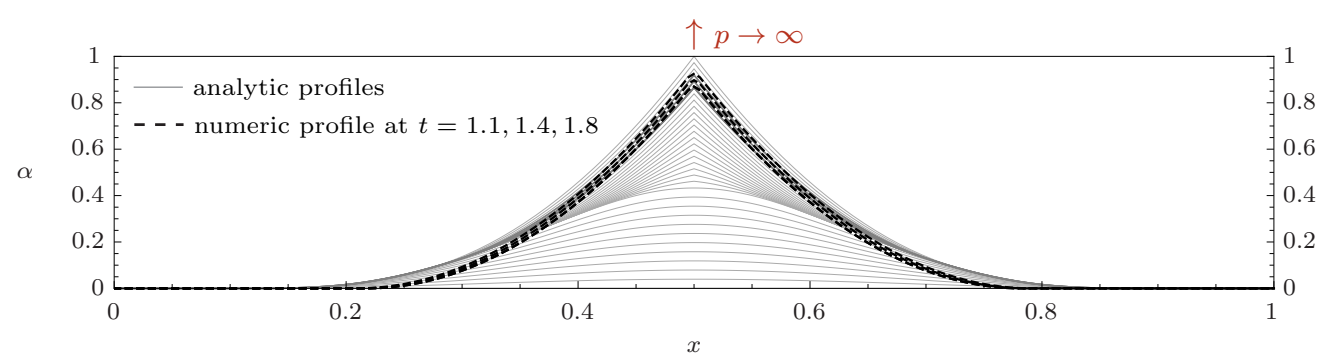

(e)

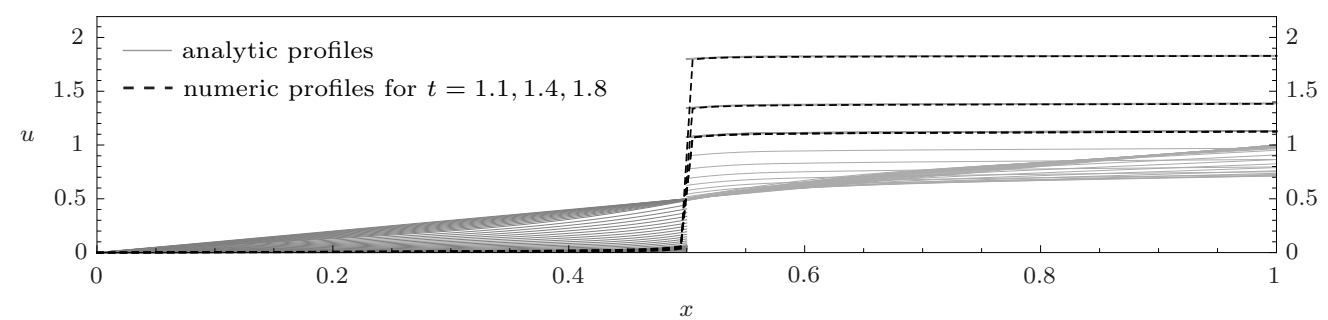

(f)

Figure 5: Numerical and analytical results for the traction test of the $E-D-P D$ model: (a) force-displacement diagram; (b) energy diagrams; (c) fracture energy-crack opening diagram; (d) stress-crack opening diagram; damage (e) and displacement (f) fields at different loading steps. The force-displacement diagram (a) compares the analytical results obtained taking into account (continuous line) or not (dashed line) the irreversibility condition. 
the total energy is not conserved when the crack is nucleated. The numerical results are in close agreement with the analytical predictions.

\subsection{E-P-D response}

In this case, after the initial elastic stage, a perfect plastic stage occurs. Hence, the plastic strains increase uniformly in space ${ }^{2}$ until the end-displacement reaches a second yield value, $t_{\mathrm{yy}}=2$ and $\sigma_{\mathrm{yy}}=1$, when the damage criterion is also triggered. For $t>t_{\mathrm{yy}}$, the structural response may be very different from the material response. In the latter, after the plastic stage, only damage evolves. Instead, in the structural response, the damage evolution is coupled to a plastic localisation. In the present onedimensional traction test, the qualitative features of the numerical response depend on the internal length. Figures $6(\ell=0.15)$ and $7(\ell=0.08)$ illustrate the two possible scenarios associated with large and small values of the internal length, respectively.

For $\ell=0.15$, from the force-displacement plot of Fig. 6a we observe that up to a very low stress level the numerical response follows the stress softening branch, without snap-back. The energy plots of Fig. 6b unveil the dissipating phenomena occurring in the softening branch, with a contemporary evolution of damage and localised plastic strain. The evolution of the damage and displacement profiles in Figures 6e and 6f show the presence of a displacement jump at the center of the damage localisation zone. This jump occurring with $\alpha<1$ is associated to a plastic singularity, as per 19. In this singular point, the damage evolution criterion (see Table 2 imposes a link between the plastic singularity and the jump in $\alpha^{\prime}$, namely $2 \mathrm{w}_{1} \ell^{2} \llbracket \alpha^{\prime} \rrbracket-\sigma_{\mathrm{P}}^{\prime}(\alpha) \bar{P}=0$. The detailed analytical calculation of the solution is reported in Appendix A. From the plot of the energy-displacement jump in Fig. 6c we recognize a Dugdale's like cohesive fracture behaviour. Differently form the case of the $E-D-P D$ model in Fig. 5 the nucleation of a displacement jump does not require a finite energy dissipation. Both the energy and the displacement jumps increase smoothly and monotonically from 0 with the loading, until the occurrence of a snap-back in the response. The corresponding cohesive force is represented in Fig. 6d, the stress decreases linearly during the plastic strain localisation.

For a smaller internal length $(\ell=0.08)$, the results of the numerical computation obtained with the alternate minimization miss the softening transition in the forcedisplacement response, Fig. 7a. All the fracture energy is instantaneously dissipated Fig. 7b. The cohesive-like effect is not visible because unstable. Numerically, in this case, a fracture occurs without the growth of the plastic localisation, but only with a pure damage localisation, reaching

\footnotetext{
${ }^{2}$ In a perfect plasticity setting, the homogeneous plastic evolution is not unique. Nevertheless, in the present one-dimensional context and for sake of simplicity, only homogeneous plastic strains are taken into account before the damage triggering.
}

immediately its maximum value at the center, like for the $E-D$ model, Fig. $7 \mathrm{c}$. After the occurrence of the fracture, that is with a vanishing stress, the elastic strain vanishes. Therefore, the total strain, related to the non vanishing slope of the displacements profiles, Fig. 7d, compensates the plastic strain formerly occurred during the evolution. The total energy is not conserved at the fracture appearance.

Despite of what is numerically observed for small internal lengths, the existence of cohesive response of the Dugdale type can be proven for any $\ell$. To this end, we can look for the existence of solutions where

$$
\bar{p}^{\mathrm{S}}=\llbracket u \rrbracket=\left(1-\frac{\sigma}{\sigma_{\mathrm{p}}}\right) u_{\mathrm{c}}
$$

at the singular point and zero elsewhere. From the damage jump condition on the singular domain, and from the damage and plastic yield criteria in Tab2, we derive the following relations in the very same singular point:

$$
\left|\alpha^{\prime}\right|=\frac{\left(\sigma_{\mathrm{p}}-\sigma\right) u_{\mathrm{c}}}{4 \mathrm{w}_{1} \ell^{2}}, \quad \bar{\alpha}=\left(1-\frac{\sigma}{\sigma_{\mathrm{p}}}\right), \quad \bar{p}^{\mathrm{R}}=\frac{\mathrm{w}_{1}}{\sigma_{\mathrm{p}}}-\mathrm{S}_{0} \sigma_{\mathrm{p}} .
$$

with $\llbracket \alpha^{\prime} \rrbracket=2\left|\alpha^{\prime}\right|$. We can also prov $\rrbracket^{3}$ that there exists a critical opening

$$
u_{\mathrm{c}}^{2}=8 \mathrm{~S}_{0} \mathrm{w}_{1} \ell^{2}
$$

for which the cohesive stress vanishes. For small values of the internal length this kind of solution is not obtained through the alternate minimization because it is unstable.

This example underlines some limitations of the alternate minimization algorithm for time-discontinuous evolutions and rises the question of the opportunity of using path-following or minimizing mouvements [26] algorithms to better describe the dissipative phenomena in unstable snap-back branches.

\subsection{E-P-PD response}

This response is similar to the $E-P-D$ model, except for the fact that during the fracturing stage, a Barenblatt's cohesive fracture behaviour is recovered. After the initial elastic stage, the same perfect plastic stage occurs, where the plastic strains increase uniformly in space. At $\left(t_{\mathrm{yy}}, \sigma_{\mathrm{yy}}\right)=(2,1)$ a cohesive fracture appears at the centre of the damage localisation zone. In the numerical response for $\ell=0.15$, the stress jumps to a value that is almost half the yield stress, as highlighted by the stress-displacement plot, Fig. 8a. The immediately contemporary evolution of

\footnotetext{
${ }^{3}$ It is sufficient to prove that for any stress level, during the evolution of a localisation, both $\left(\alpha, \alpha^{\prime}\right)$ given by 28) and 290 satisfy A.34, where the constant is obtained by assuming that A.34 is satisfied also for $\left(\alpha, \alpha^{\prime}\right)=(0,0)$. This gives

$$
C=-\frac{1}{2} \mathrm{~S}_{0} \sigma^{2}+\mathrm{w}_{1}-\mathrm{S}_{0} \sigma_{\mathrm{p}}^{2}
$$

Injecting now 28, 29, and 30 in A.34 we obtain the expression
} for $u_{c}$. 


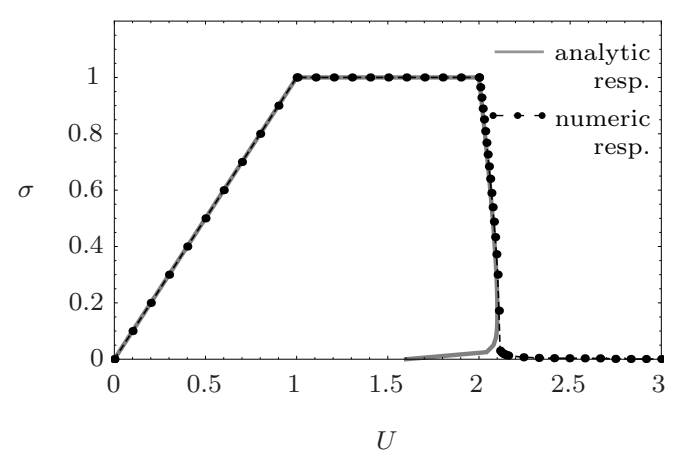

(a)

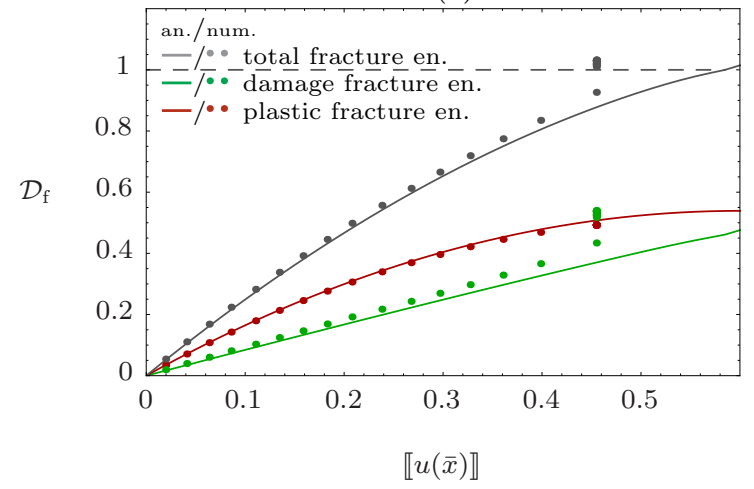

(c)

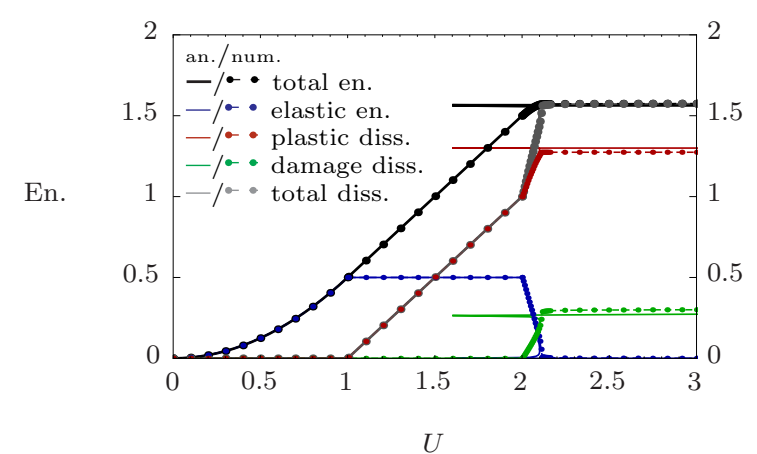

(b)

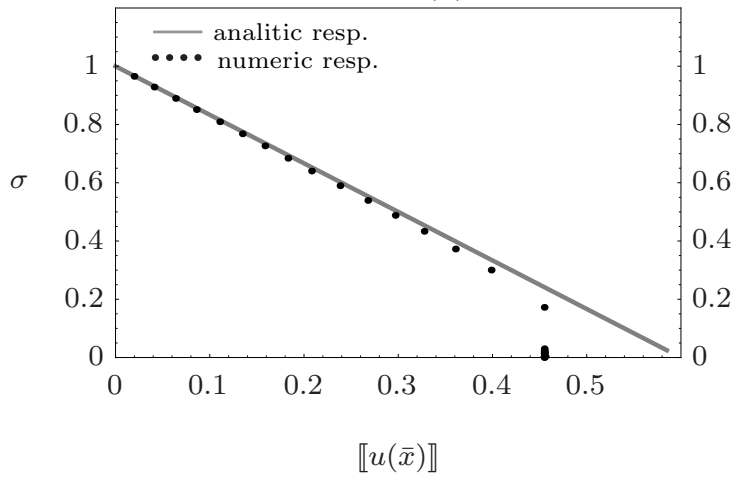

(d)

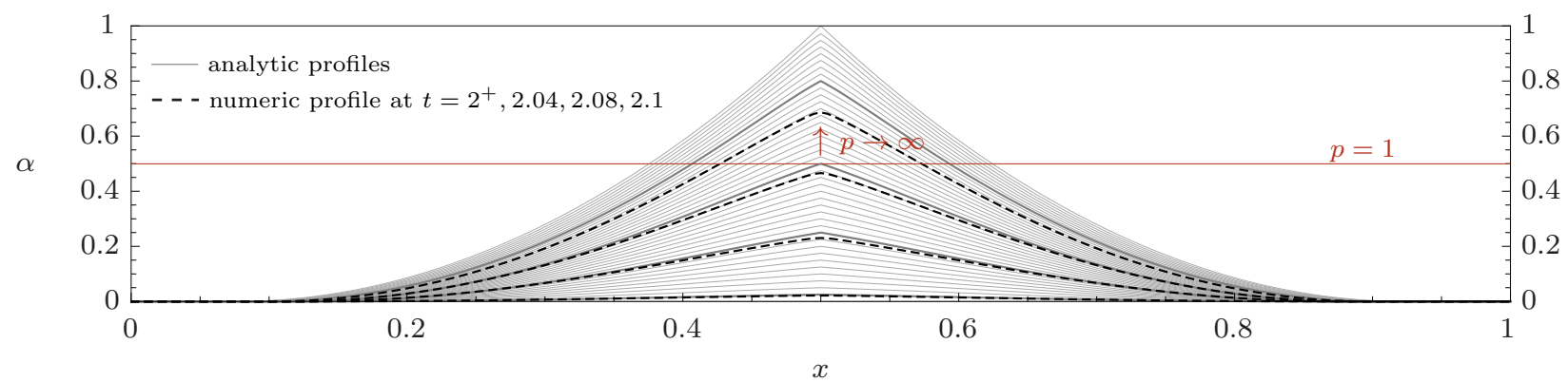

(e)

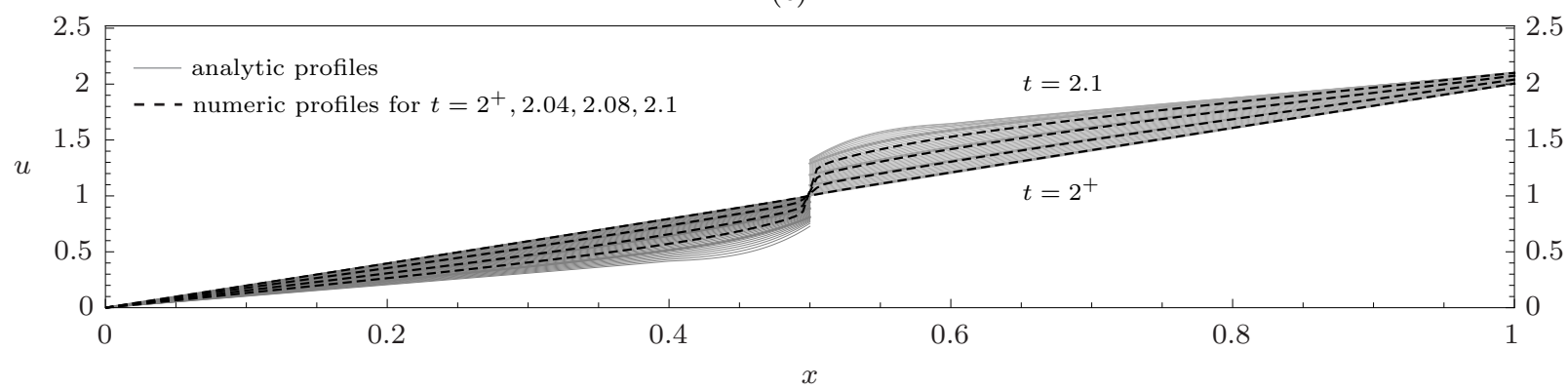

(f)

Figure 6: Numerical and analytical results for the traction test of the $E-P-D$ model with $\ell=0.15$ : (a) force-displacement diagram; (b) energy diagrams; (c) fracture energy-crack opening diagram; (d) stress-crack opening diagram; damage (e) and displacement (f) fields at different loading steps. 


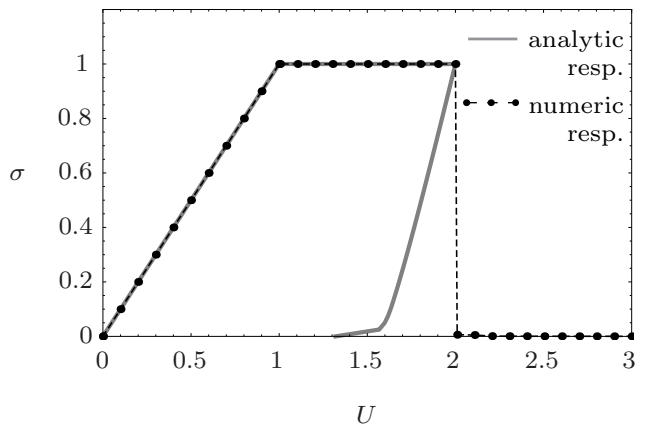

(a)

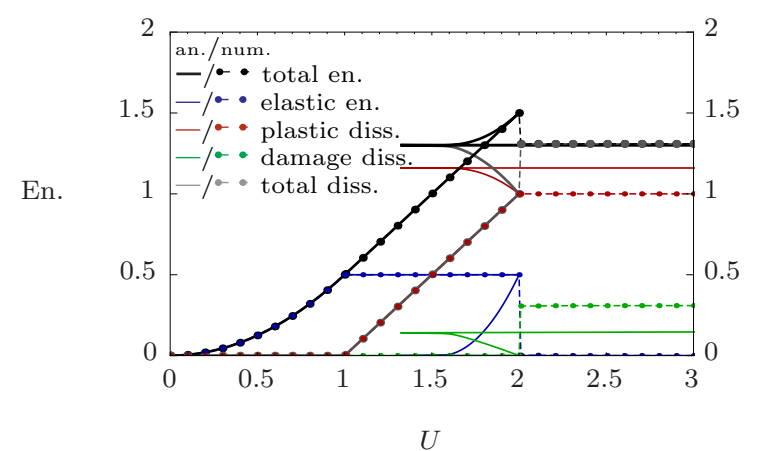

(b)

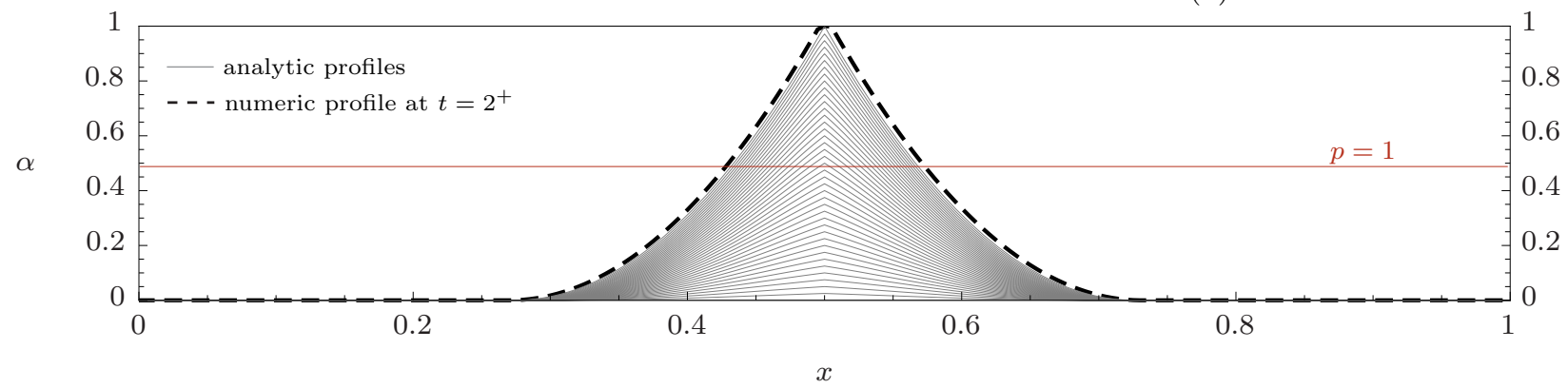

(c)

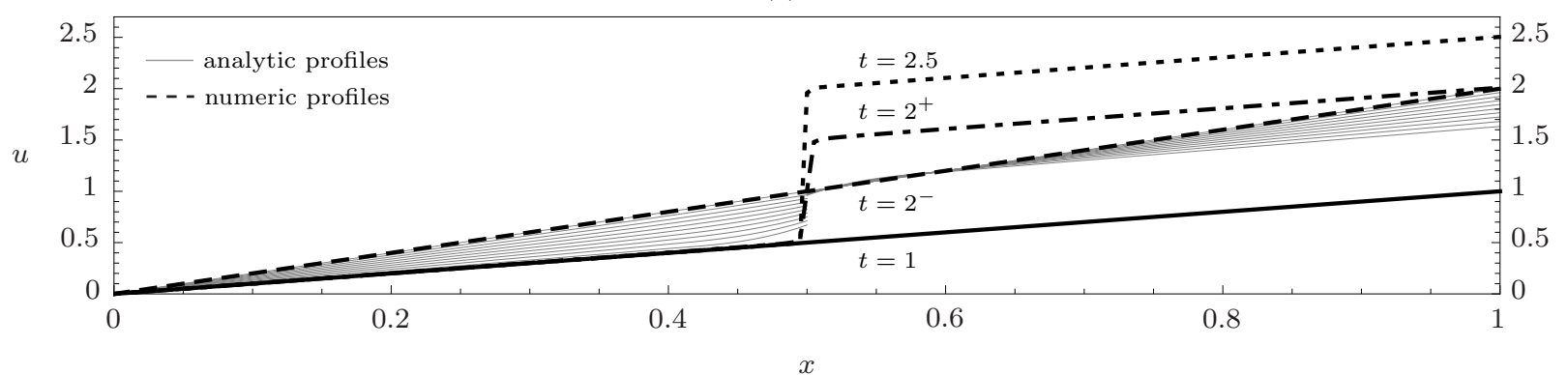

(d)

Figure 7: Numerical and analytical results for the traction test of the $E-P-D$ model with $\ell=0.08$ : (a) force-displacement diagram; (b) energy diagrams; (c) fracture energy-crack opening diagram; (d) stress-crack opening diagram; damage (e) and displacement (f) fields at different loading steps. 
damage and plasticity during the fracturing stage is, instead, evident from the energy plot in Fig. 8b. The resulting fracture behaviour can be identified as a Barenblattlike cohesive fracture response, simply by looking at the fracture energy contribution with respect to the fracture opening displacement, Fig. 8c The interfacial stress law with respect to the crack opening is plotted in Fig. 8d, After the initial jump, the stress monotonically decreases, approaching asymptotically zero only for an infinite crack opening. The capability of such model in describing Barenblatt's cohesive fracture behaviour has been already and rigorously proved in [5] with closed form results for a model with ad-hoc constitutive functions, different from the ones considered here. Note that numerical algorithm based on alternate minimization is not able to follow the unstable part of the localised solution branch, associated with a snap-back. Fig. 9 reports the stress-strain responses obtained for several values of $\ell$, emphasising the role of the internal length on the snap-back. The damage profiles, built using the damage localisation profiles obtained in the appendix, evolve from the very beginning with a cusp at the center of the localisation, Fig. 8e, due to the contemporary evolution of a plastic localisation in the same point. Therefore, the displacement field suffers a sharp discontinuity during the crack opening which is well captured by the numerical model, Fig. $8 \mathrm{8f}$ Also for the present case, the total energy is not conserved at the fracture appearance.

In the $E-P_{-}{ }^{*}$ models, the amount of plastic strain, accumulated before the triggering of damage, has a key role in the localisation and structural responses. Fig. 10 highlights the force-displacement response for the $E-P$ $P D$ model and for different values of the critical accumulated plastic strain $\bar{p}_{c}$, that is, the accumulated plastic strain at which damage is triggered and given by the relation $\mathrm{w}_{1}=2 \bar{p}_{c}$. By increasing $\mathrm{w}_{1}$, one increases the initial damage yield stress 24 and, therefore, postpones the triggering of the localisation. We note then, that the more the plastic strain is accumulated before damage is triggered, the less the snap-back phenomenon is accentuated. Moreover, from a certain value, the snap-back phenomenon disappears in favour of a smooth stress-softening branch. This effect is due to a progressive widening of the localisation support, as the one observed when increasing the internal length.

\section{Conclusions and Perspectives}

We have studied the complex response of nonlinear continuum models including coupled damage and plasticity, with the aim of developing an effective phase-field model of ductile and cohesive fracture. The model has been presented in a rigorous variational framework consistent with the theory of Generalised Standard Materials. It includes a localisation limiter only on the damage variable (damagegradient models). We expanded here the ideas presented in a previous work [5, by analysing the response of a one-dimensional bar when using the classes of constitutive

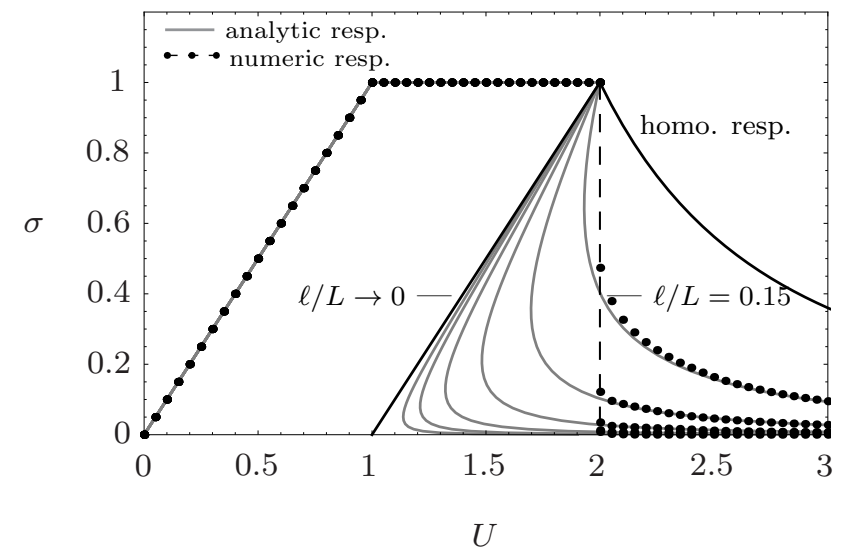

Figure 9: Influence of the internal length on the force-displacement diagram for the $E-P-P D$ model. The different curves are for $\ell / L=$ $\{0.1500,0.0750,0.0375,0.0188,0.0094,0.0047\}$. The points denotes the solutions obtained numerically through the alternate minimization, which does not follow the unstable parts of the branches.

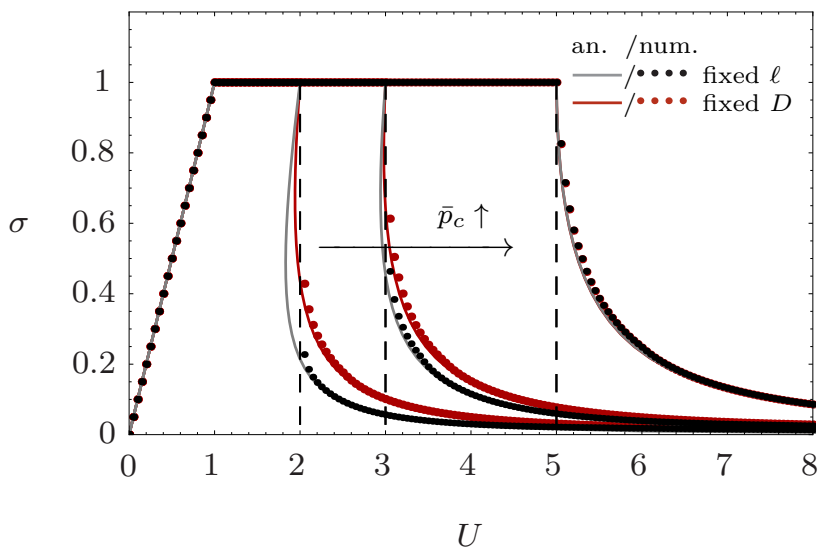

Figure 10: Force-displacement diagram for the $E-P-P D$ model for different values of the critical accumulated plastic strain $\left(\bar{p}_{c}=\right.$ $\{1,2,4\})$ corresponding, respectively, to $w_{1}=\{3,5,9\}:(-) /(\cdots \cdots)$ correspond to analytical/numerical responses for a fixed internal length, $\ell=0.11 ;(-) /(\cdots)$ correspond, instead, to the analytical/numerical responses for different internal lengths, $\ell=$ $\{0.155,0.131,0.110\}$, respectively, in order to keep fixed the dimension of the localisation support.

functions usually employed in numerical phase-field models of fracture. We presented a throughout comparison of the different macroscopic responses that can be obtained by semi-analytical solutions and numerical time-discrete alternate minimisation algorithms. We have shown that the interplay between damage and plasticity can lead to a rich macroscopic behaviour, depending of the ratio of the yield stresses and softening properties of the plasticity and damage yield criteria. We have analysed the responses obtained for four different regimes, leading to macroscopic behaviour associable to brittle fracture à la Griffith $(E$ $D$ model), cohesive fracture of the Barenblatt (E-P-PD model) or Dugdale type $(E-P-D)$, and a kind of cohesive fracture including a depinning energy contribution $(E-D$ $P D$ model). A key feature of the evolution is the subtle coupling between fully localised plastic deformations and 
(a)

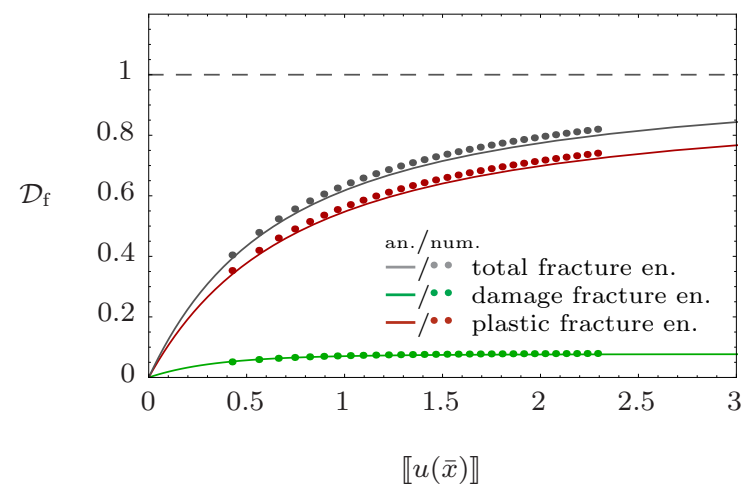

(c)
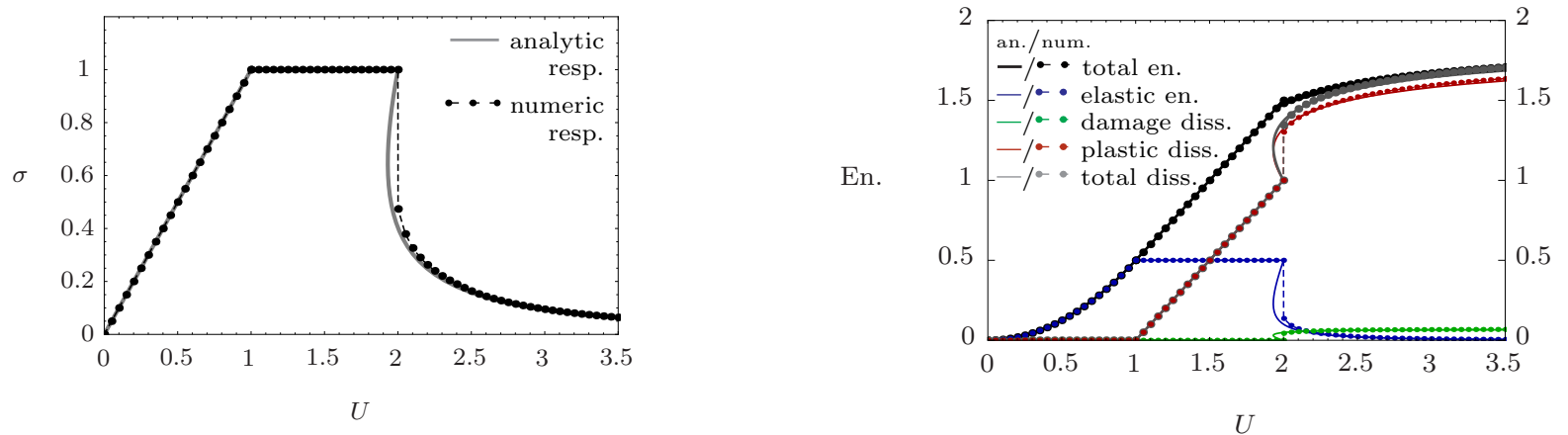

(b)

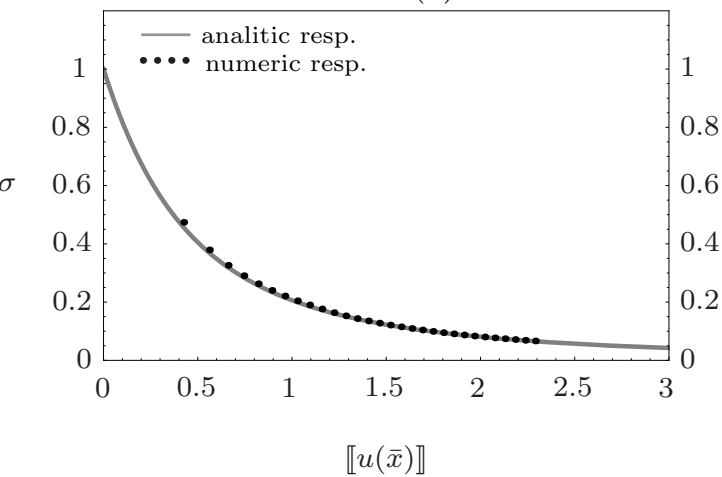

(d)

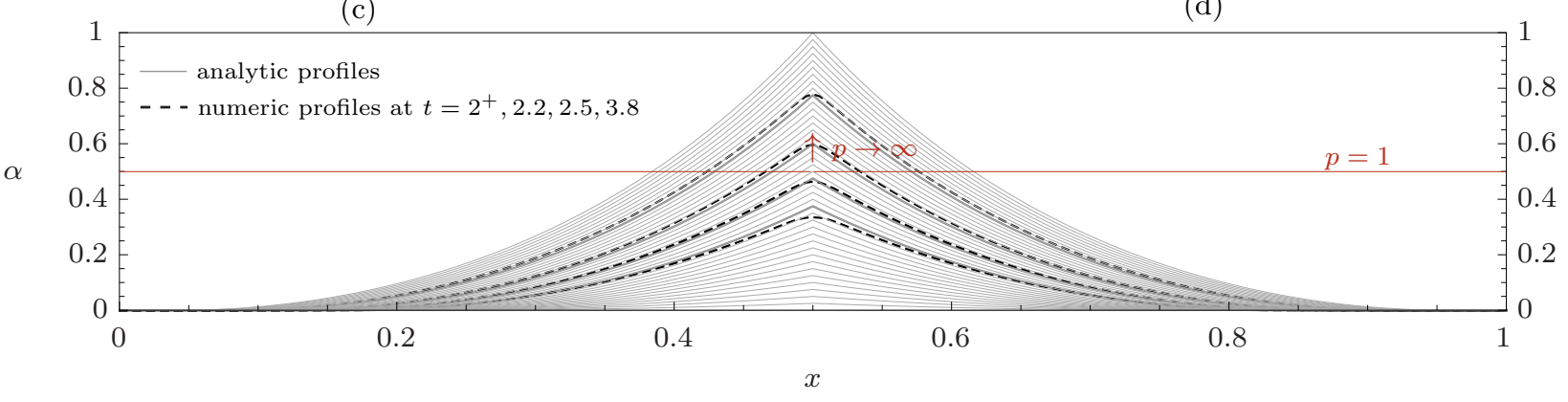

(e)

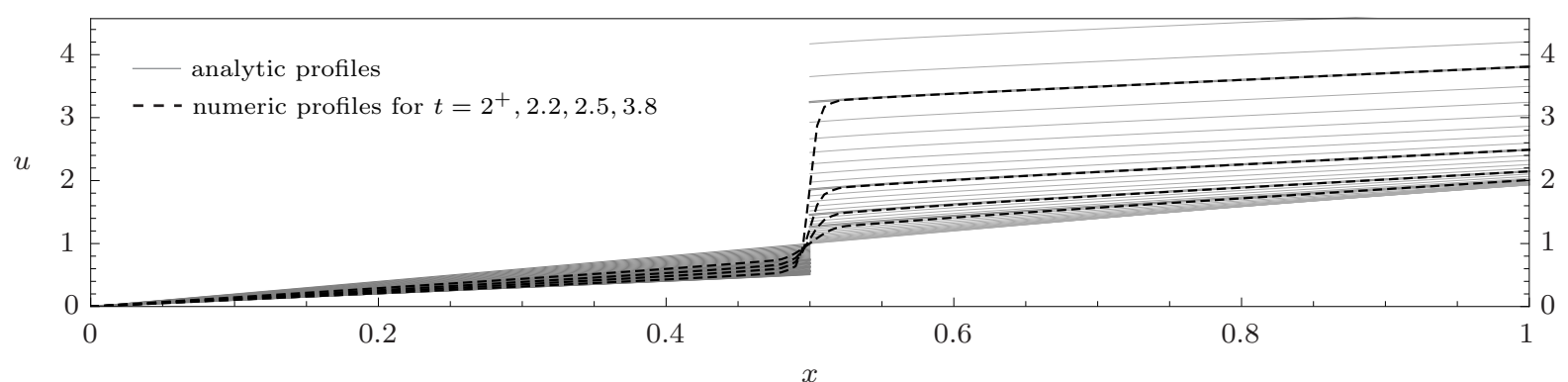

(f)

Figure 8: Numerical and analytical results for the traction test of the $E-P-P D$ model: (a) force-displacement diagram; (b) energy diagrams; (c) fracture energy-crack opening diagram; (d) stress-crack opening diagram; Damage (e) and displacement (f) fields at different loading steps. 
damage localisations.

We proposed a numerical solution scheme based on alternate minimisation and reported extensive comparisons with semi-analytical results in the different regimes. The agreement between numerical and analytical results is very good in most cases. However, our analysis emphasises that issues can appear in time-discontinuous evolutions, as encountered when a crack suddenly nucleates. These events are associated to the loose of stability of fundamental solutions branches and the simultaneous appearance of bifurcated branches, which are often unstable. The numerical responses obtained with the alternate minimisation algorithm jumps to new stable solutions, without preserving the total energy and without accounting for irreversible evolutions during the jumps. The solutions obtained analytically, by following the unstable branches emerging at the instability of the fundamental branch, may be different. This is not surprising since the evolution problem has multiple solutions and one can expect that different algorithms can lead to different solutions. However, this poses the general problem of the selection of the better algorithm to give a physically pertinent solution. We do not have an answer to this fundamental question.

Another interesting feature of the present model, not explored in this work, is the capability to describe plastic fatigue effects, 32 .

We believe that our contribution provide a better insight in phase-field approaches to ductile and cohesive fracture and can help in improving the currently available models. Our work focused on the one-dimensional traction test. The extension to two- and three-dimensional contexts is currently under investigation.

\section{Appendix A. Construction of damage localization profiles in the $1 \mathrm{D}$ setting}

When the domain is assumed to be a line segment $[0, L]$, a procedure for the construction of a single localization zone is shown for several different choices of the constitutive parameters; these choices reflect the order with which plasticity and damage occur, according to Tab. 3 The damage profiles constructed will allow to obtain the global response.

The attention is here limited on the formation and evolution of only one internal localized damaged zone, $\mathcal{S}=$ $[\bar{x}-D, \bar{x}+D]$ where $D$ and $\bar{x}$ denote respectively half its size and the position of its center. Therefore we exclude from the analysis damage localizations near the boundaries and the interactions between multiple damage profiles. Obviously, with respect to the homogeneous cases, Sec. 3 the damage gradient term plays a crucial role since the damage yield condition becomes a differential equation in $\alpha$ from which the damage profile is constructed.

We recall from Tab. 2 the plastic and damage yield functions:

$$
\begin{aligned}
f_{\mathrm{p}}(\sigma, \alpha) & =f_{\mathrm{p}}^{\mathrm{S}}(\sigma, \alpha)=|\sigma|-\sigma_{\mathrm{P}}(\alpha), \\
f_{\mathrm{d}}\left(\sigma, \bar{p}^{\mathrm{R}}, \alpha\right) & =\frac{1}{2} \mathrm{~S}^{\prime}(\alpha) \sigma^{2}-\mathrm{w}^{\prime}(\alpha)-\sigma_{\mathrm{P}}^{\prime}(\alpha) \bar{p}^{\mathrm{R}}+2 \mathrm{w}_{1} \ell^{2} \alpha^{\prime \prime}, \\
f_{\mathrm{d}}^{\mathrm{S}}\left(\sigma, \bar{p}^{\mathrm{S}}, \alpha\right) & =2 \mathrm{w}_{1} \ell^{2} \llbracket \alpha^{\prime} \rrbracket-\sigma_{\mathrm{P}}^{\prime}(\alpha) \bar{p}^{\mathrm{S}},
\end{aligned}
$$

defining the plastic yield stress $\sigma_{\mathrm{P}}(\alpha)$ and the damage yield stress

$$
\sigma_{\mathrm{D}}\left(\bar{p}^{\mathrm{R}}, \alpha\right):=\sqrt{\frac{2\left(\mathrm{w}^{\prime}(\alpha)+\sigma_{\mathrm{P}}^{\prime}(\alpha) \bar{p}-2 \mathrm{w}_{1} \ell^{2} \alpha^{\prime \prime}\right)}{\mathrm{S}^{\prime}(\alpha)}} .
$$

If $\sigma_{\mathrm{P}}(0)>\sigma_{\mathrm{D}}(0,0)$, a $E-D-^{*}$ response occurs, otherwise we get a $E-P_{-}{ }^{*}$ response.

The construction of damage profile starts when the damage yield criterion is satisfied somewhere in the bar as an equality. This instant corresponds either to the end of an elastic stage, if $\sigma_{\mathrm{D}}(0,0)<\sigma_{\mathrm{P}}(0)$, or at the end of a plastic stage, if $\sigma_{\mathrm{D}}(0,0)>\sigma_{\mathrm{P}}(0)$. When damage is triggered, the stress $\sigma$, constant along the bar, monotonically decreases to 0 from $\sigma_{\mathrm{y}}:=\min \left\{\sigma_{\mathrm{P}}(0), \sigma_{\mathrm{D}}(0,0)\right\}$, due to the softening conditions, (6). Starting from an undamaged state, the purpose is to determine the damaged localization profile $\alpha(x)$ and the corresponding plastic strain fields $p(x)$ and $\bar{p}(x)$ for any stress level $\sigma \in\left[0, \sigma_{\mathrm{y}}\right]$. In particular:

1. A homogeneous undamaged state, where $\sigma=\sigma_{\mathrm{y}}$, is chosen to start with;

2. The damage yield condition $f_{\mathrm{d}}=0$, a differential equation in $\alpha$, is initially used to determine the damage profile as $\sigma$ is decreased. At this stage, no singular points are assumed, hence $J(\boldsymbol{\xi})=\emptyset$. This analysis produces a function $\sigma \mapsto \alpha_{\sigma}(x)$, mapping any stress level in the damage profile at constant plastic strain, $\dot{\bar{p}}=0$;

3. The violation of the plastic yield condition $f_{\mathrm{p}}=0$ is checked for all $\sigma$. A violation may occur if in some points $\sigma>\sigma_{\mathrm{P}}\left(\alpha_{\sigma}\right)$. In this case, singular points may appear, and also the singular damage yield criterion A.3 must be taken into account.

In all the steps, the damage irreversibility condition is explicitly considered.

\section{Appendix A.1. E-D-* case}

The following analysis has strong analogies with the work in [57. The reader is therefore invited to refer therein for additional details.

The instant where a localization may appear corresponds to the end of the elastic phase: this implies that plastic strains are vanishing everywhere, that no singular 
points are present and that $\sigma=\sigma_{\mathrm{y}}=\sigma_{\mathrm{D}}(0,0)$. The starting state for the construction of a localisation is, then,

$$
(u, p, \bar{p}, \alpha)(x)=\left(\left(\sigma_{\mathrm{y}} / \mathrm{E}_{0}\right) x, 0,0,0\right) .
$$

The localization process is then governed only by the damage yield criterion,

$$
-\frac{1}{2} \mathrm{~S}^{\prime}(\alpha) \sigma^{2}+\mathrm{w}^{\prime}(\alpha)-2 \mathrm{w}_{1} \ell^{2} \alpha^{\prime \prime}=0, \quad \forall x \text { in } \mathcal{S} .
$$

This last autonomous second order differential equation is an Euler-Lagrange equation which admits a first integral,

$$
-\frac{1}{2} \mathrm{~S}(\alpha) \sigma^{2}+\mathrm{w}(\alpha)-\mathrm{w}_{1} \ell^{2} \alpha^{\prime 2}=C, \quad \forall x \text { in } \mathcal{S}
$$

The constant $C$ is determined by the boundary conditions which depends on the irreversibility condition. In the most general case, one has

$$
\mathrm{w}_{1} \ell^{2} \alpha^{\prime}(x)^{2}=\mathrm{H}(\sigma, \alpha(x), C), \quad \forall x \text { in } \mathcal{S},
$$

with

$$
\mathrm{H}(\sigma, \alpha, C):=-\frac{1}{2} \mathrm{~S}(\alpha) \sigma^{2}+\mathrm{w}(\alpha)-C .
$$

Taking advantage of the matching conditions of the damage profile with the elastic zone

$$
\alpha(\bar{x} \pm D)=\alpha^{\prime}(\bar{x} \pm D)=0
$$

we obtain

$$
C(\sigma)=-\frac{1}{2} \mathrm{~S}_{0} \sigma^{2}+\mathrm{w}(0)
$$

The function $\mathrm{H}(\sigma, \alpha, C(\sigma))$ vanishes for $\alpha=0$ and $\alpha=\alpha^{*}(\sigma)$ with $0<\alpha^{*}(\sigma)<1$, [57. Moreover, the damage profile has a maximum in $\bar{x}$ such that $\alpha(\bar{x})=\alpha^{*}(\sigma)$ and is symmetric, hence $\alpha^{\prime}(\bar{x})=0$. It is then possible to construct the damage profile in the localization by using

$$
\sqrt{\mathrm{w}_{1}} \ell \alpha^{\prime}=\operatorname{sign}(x-\bar{x}) \sqrt{\mathrm{H}(\sigma, \alpha, C(\sigma))}, \quad \forall x \text { in } \mathcal{S} .
$$

By separating the variables $x$ and $\alpha$ in A.12, one gets the damage field $\alpha_{\sigma}(x)$ in an implicit form,

$$
x-\bar{x}=\sqrt{\mathrm{w}_{1}} \ell \int_{\alpha}^{\alpha^{*}} \frac{1}{\sqrt{\mathrm{H}(\sigma, \beta, C(\sigma))}} \mathrm{d} \beta .
$$

Thus one has determined the damage field with respect to the stress level. The half-damage support $D$ becomes a function of $\sigma$ and from A.36 it reads

$$
D(\sigma)=\sqrt{\mathrm{w}_{1}} \ell \int_{0}^{\alpha^{*}} \frac{1}{\sqrt{\mathrm{H}(\sigma, \beta, C(\sigma))}} \mathrm{d} \beta,
$$

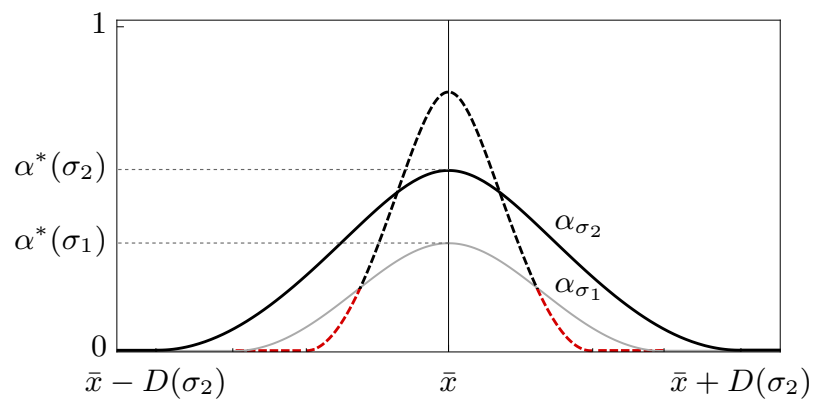

Figure A.11: Admissible and not-admissible evolutions for the damage profile. The black, thick curve represents an admissible evolution while the dashed curve violates the irreversibility condition in the red part and hence represents a not admissible evolution. The solid gray curve represents a damage profile at a previous time instant.

with the limit case $\sigma=0$ simply given by

$$
D(0)=\sqrt{\mathrm{w}_{1}} \ell \int_{0}^{1} \frac{\mathrm{d} \alpha}{\sqrt{\mathrm{w}(\alpha)}} .
$$

The issue is now, whether the constructed map $\sigma \rightarrow$ $\alpha_{\sigma}(x)$ satisfies at any point $x \in \mathcal{S}$ the irreversibility condition or not. Specifically:

1. if for any $\sigma$, the damage field $\sigma \rightarrow \alpha_{\sigma}(x)$ satisfies the irreversibility condition, that is

$$
\forall \sigma_{2}<\sigma_{1} \text { and } \forall x \in \mathcal{S}_{\sigma_{1}}, \Rightarrow \alpha_{\sigma_{2}}(x) \geq \alpha_{\sigma_{1}}(x)
$$

the construction is admissible. In this last relation $\mathcal{S}_{\sigma_{1}}=\left(\bar{x}-D\left(\sigma_{1}\right), \bar{x}+D\left(\sigma_{1}\right)\right)$;

2. on the contrary, if a $\sigma_{2}<\sigma_{1}$ exists such that in a subset of $\mathcal{S}$ the damage field decreases, that is

$\exists \sigma_{2}<\sigma_{1}$ and for some $x \in \mathcal{S}_{\sigma_{1}}, \Rightarrow \alpha_{\sigma_{2}}(x)<\alpha_{\sigma_{1}}(x)$

the solution is not admissible and a different construction is proposed.

Both these situations are represented in Fig. A.11.

When the irreversibility condition is violated, the damage field continues to evolve only in a subset $\mathcal{S} \supset \hat{\mathcal{S}}=$ $(\bar{x}-\hat{D}, \bar{x}+\hat{D})$, with $\hat{D}<D\left(\sigma_{0}\right)$, while the complementary domain is subjected to an elastic unloading. Within $\hat{\mathcal{S}}$, the governing equation is always A.8, but a different constant $C$ has to be found using the continuity of $\alpha(x)$ and $\alpha^{\prime}(x)$ in $x=\bar{x} \pm \hat{D}$.

The new constant $\hat{C}$ is given by

$\hat{C}(\sigma, \hat{D})=-\frac{1}{2} \mathrm{~S}\left(\hat{\alpha}_{0}(\bar{x}-\hat{D})\right) \sigma^{2}+\mathrm{w}\left(\hat{\alpha}_{0}(\bar{x}-\hat{D})\right)-\mathrm{w}_{1} \ell^{2} \hat{\alpha}_{0}^{\prime \prime}(\bar{x}-\hat{D})$,

so that the new maximum damage level is

$$
\alpha^{*}(\sigma, \hat{D}) \text { such that } \mathrm{H}\left(\sigma, \alpha^{*}, \hat{C}(\sigma, \hat{D})\right)=0 \text {. }
$$




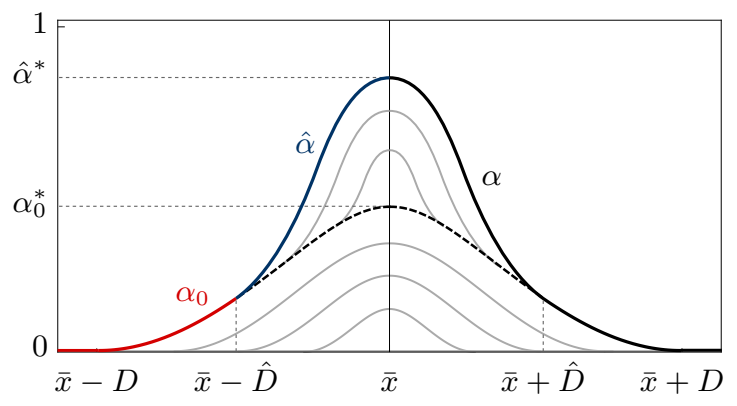

Figure A.12: The solid black thick curve represents the modified construction that satisfies irreversibility. The solid red branch is the profile which does not evolve because subjected to an elastic unloading while the blue branch represents an evolving profile. The solid gray curves represent damage profiles at previous time instants.

The unknown length $\hat{D}$ is implicitly given by

$$
\hat{D}-\bar{x}=\sqrt{\mathrm{w}_{1}} \ell \int_{\alpha_{0}(\bar{x}-\hat{D})}^{\alpha^{*}(\sigma, \hat{D})} \frac{\mathrm{d} \beta}{\sqrt{\mathrm{H}(\sigma, \beta, \hat{C}(\sigma, \hat{D}))}},
$$

where $\alpha_{0}$ is the last damage profile satisfying irreversibility. Most of the time, this last relation cannot be resolved explicitly and a trial-error numeric scheme has to be adopted. A numeric implementation has been carried out with the software Mathematica. Once the new damage support $\hat{D}$ has been found, the damage profile, in the evolving domain $\hat{\mathcal{S}}$, is given by the implicit relation

$$
\hat{\alpha}_{\sigma}(x): x-\bar{x}=\sqrt{\mathrm{w}_{1}} \ell \int_{\hat{\alpha}}^{\alpha^{*}(\sigma, \hat{D})} \frac{1}{\sqrt{\mathrm{H}(\sigma, \beta, \hat{C}(\sigma))}} \mathrm{d} \beta,
$$

that combined with the damage profile $\alpha_{0}$, subjected to the elastic unloading, gives the overall damage field evolution,

$$
\alpha_{\sigma}(x)= \begin{cases}\hat{\alpha}_{\sigma}(x), & \forall x \in \hat{\mathcal{S}} \\ \alpha_{0}(x), & \forall x \in \mathcal{S} \backslash \hat{\mathcal{S}}\end{cases}
$$

This last construction is depicted in Fig. A.12,

Now that one is able to construct damage localization evolutions, which satisfy the irreversibility condition and are based on the first order stability condition, another question arises. Namely if during such evolution $\sigma \rightarrow \alpha_{\sigma}$ the plastic yield criterion is violated or not. Two cases are possible:

1. During all the evolution the plastic criterion is never violated,

$$
\sigma<\sigma_{\mathrm{P}}\left(\alpha_{\sigma}(x)\right), \quad \forall x \in \mathcal{S}
$$

2. During the evolution it exist a stress level $\sigma$ and at least one point $x$ where the plastic yield condition is violated,

$$
\exists x \in \mathcal{S}, \sigma>0, \quad \text { such that } \sigma \geq \sigma_{\mathrm{P}}\left(\alpha_{\sigma}(x)\right) .
$$

In case 1 , the solution is admissible and the damage process becomes a candidate for the global response since it fulfills all the requirements of the first order stability. Therefore, the above construction is able to describe localizations for a $E-D$ response.

On the other hand, case 2 refers to a process in which the plastic criterion is attained. Such situation is hereafter described.

$E-D-P D$ response. The plastic criterion can be violated only at one point $x=\bar{x}$, namely at center of the damage profile where $\alpha$ is maximum and, therefore, $\sigma_{\mathrm{P}}(\alpha)$ minimum, (6).

The critical stress level, where the plastic criterion is violated, is $\bar{\sigma}=\sigma_{\mathrm{P}}\left(\alpha_{\sigma}(\bar{x})\right)$. This means that in the construction of a localization the maximum value of the damage field is not anymore dictated by the condition $\alpha^{\prime}(\bar{x})=0$ where $\mathrm{H}=0$ but by

$$
\bar{\alpha}(\sigma):=\alpha_{\sigma}(\bar{x})=\sigma_{\mathrm{P}}^{-1}(\sigma) .
$$

Moreover, due to (A.3), the derivative of the damage profile must suffer a jump in the very same point. The center of the localization becomes, then, for any $\sigma \leq \bar{\sigma}$, a singular point $J(\boldsymbol{\xi})=\{\bar{x}\}$. Since the damage profile in the connected sub-regions $[0, L] \backslash J(\boldsymbol{\xi})$ is still governed by A.6 and the accumulated plastic strain is zero in $\mathcal{S} \backslash\{\bar{x}\}$, the damage field reads

$$
x-\bar{x}=\sqrt{\mathrm{w}_{1}} \ell \int_{\alpha}^{\bar{\alpha}(\sigma)} \frac{1}{\sqrt{\mathrm{H}(\sigma, \beta, C(\sigma))}} \mathrm{d} \beta,
$$

where $\bar{\alpha}$ is given by A.25). Clearly, the damage profile is still symmetric with respect to the center $\bar{x}$ but its derivative is not anymore continuous. A jump $\llbracket \alpha^{\prime} \rrbracket$ occurs in $\bar{x}$ and its value, according to A.8, reads

$$
\llbracket \alpha^{\prime} \rrbracket(\bar{x})=-\frac{2}{\sqrt{\mathrm{w}_{1} \ell}} \sqrt{\mathrm{H}\left(\sigma, \alpha^{*}, C(\sigma)\right)},
$$

from which one deduces, with the singular plastic yield condition A.3, the coefficient 19 of the accumulated plastic strain Dirac measure

$$
\bar{p}^{\mathrm{S}}(\bar{x})=\frac{2 \mathrm{w}_{1} \ell^{2} \llbracket \alpha^{\prime} \rrbracket(\bar{x})}{\sigma_{\mathrm{P}}^{\prime}(\alpha)} .
$$

In the most general case, where irreversibility has to be taken into account, the problem becomes, for an assigned stress $\sigma<\bar{\sigma}$, to find $\hat{D}$, given by the implicit relation

$$
\hat{D}-\bar{x}=\sqrt{\mathrm{w}_{1}} \ell \int_{\alpha(\bar{x}-\hat{D})}^{\bar{\alpha}} \frac{\mathrm{d} \beta}{\sqrt{\mathrm{H}(\sigma, \beta, \hat{C}(\sigma, \hat{D}))}} .
$$




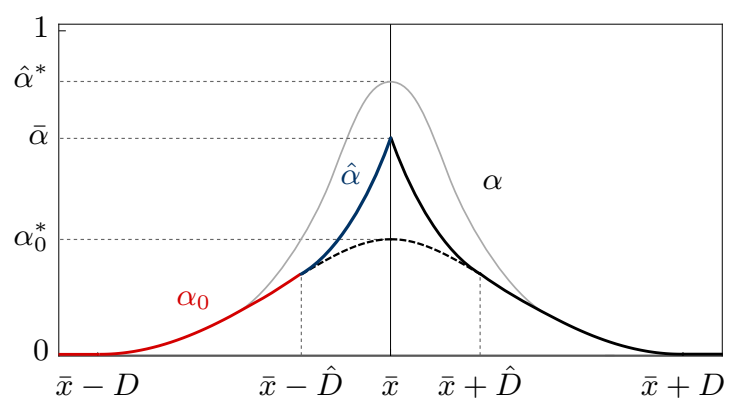

Figure A.13: The solid, black, thick curve represents the modified construction that satisfies irreversibility. The solid red branch is the profile which does not evolve because subjected to an elastic unloading while the blue branch represents an evolving profile with a jump in its derivative in $\bar{x}$. The solid gray curve represents the damage profile without plastic strains.

with the same constant $\hat{C}(\sigma, \hat{D})$ given in A.18 and the same solution strategy adopted for A.20.

The damage profile in $\hat{\mathcal{S}}$ is, in this case,

$$
\hat{\alpha}_{\sigma}(x): x-\bar{x}=\sqrt{\mathrm{w}_{1}} \ell \int_{\hat{\alpha}}^{\bar{\alpha}} \frac{1}{\sqrt{\mathrm{H}(\sigma, \beta, \hat{C})}} \mathrm{d} \beta,
$$

and the overall damage function reads

$$
\alpha_{\sigma}(x)= \begin{cases}\hat{\alpha}_{\sigma}(x), & \forall x \in \hat{\mathcal{S}} \\ \alpha_{0}(x), & \forall x \in \mathcal{S} \backslash \hat{\mathcal{S}}\end{cases}
$$

The damage construction for a $E-D-P D$ evolution is shown in Fig. A.13.

\section{Appendix A.2. E-P-* case}

In this section, we investigate the case where plasticity is triggered after the elastic stage, namely $\sigma_{\mathrm{y}}=\sigma_{\mathrm{P}}(0)<$ $\sigma_{\mathrm{D}}(0,0)$. For sake of simplicity, it is assumed that plasticity evolves uniformly along the bar.

Since $\bar{p} \mapsto \sigma_{\mathrm{D}}(\alpha, \bar{p})$ is a decreasing function with respect to plasticity and $\lim _{\bar{p} \rightarrow \infty} \sigma_{\mathrm{D}}(\alpha, \bar{p})=-\infty$, it exists, necessarily, a second yield instant where the damage criterion is attained and damage must evolve. Two possible evolutions could be expected: an $E-P-D$ response, where plasticity stops to evolve and only damage localizes, or an $E-P-P D$ response, where both damage and plasticity evolve together. Which evolution will actually take place depends on the constitutive functions. In the following, both cases are investigated and the relative localization constructions explained. Moreover, it is assumed that once a response is triggered, it persists during all the evolution, that is alternate phases of a pure damage evolution and coupled plasticity-damage evolutions are excluded. For both cases, the start for the construction of localized solutions is the homogeneous state

$u(x)=\frac{\sigma_{\mathrm{y}}}{\mathrm{E}_{0}} x, \quad p(x)=\bar{p}(x)=\frac{\mathrm{S}^{\prime}(0) \sigma_{\mathrm{y}}^{2}-2 \mathrm{w}^{\prime}(0)}{2 \sigma_{\mathrm{P}}^{\prime}(0)}, \quad \alpha(x)=0$.
E-P-D case. In this case, during the localization process, plasticity stops and does not evolve anymore. No singular points arises, $J(\boldsymbol{\xi})=\emptyset$, and the damage evolution is dictated, as for the $E-D$ response, by the damage yield criterion A.6 with the additional contribution of an initial uniform plastic distribution,

$$
-\frac{1}{2} \mathrm{~S}^{\prime}(\alpha) \sigma^{2}+\mathrm{w}^{\prime}(\alpha)+\bar{p}^{\mathrm{R}} \sigma_{\mathrm{P}}^{\prime}(\alpha)-2 \mathrm{w}_{1} \ell^{2} \alpha^{\prime \prime}=0, \quad \forall x \text { in } \mathcal{S},
$$

From now on, the procedure for the construction of the damage profiles is the same as Appendix A.1 except for the presence of the additional term $\bar{p}^{\mathrm{R}} \sigma_{\mathrm{P}}^{\prime}(\alpha)$. The first integral A.7) changes into

$$
-\frac{1}{2} \mathrm{~S}(\alpha) \sigma^{2}+\mathrm{w}(\alpha)+\bar{p}^{\mathrm{R}} \sigma_{\mathrm{P}}(\alpha)-\mathrm{w}_{1} \ell^{2}\left(\alpha^{\prime}\right)^{2}=C, \quad \forall x \text { in } \mathcal{S},
$$

while the function $\mathrm{H}$ in A.8 becomes also a function of the accumulated plastic strain, namely

$$
\mathrm{H}(\sigma, \bar{p}, \alpha, C):=-\frac{1}{2} \mathrm{~S}(\alpha) \sigma^{2}+\mathrm{w}(\alpha)+\bar{p} \sigma_{\mathrm{P}}(\alpha)-C .
$$

With such a change, the damage field $\alpha_{\sigma}(x)$ is now given by the implicit form

$$
x-\bar{x}=\sqrt{\mathrm{w}_{1}} \ell \int_{\alpha}^{\alpha^{*}} \frac{1}{\sqrt{\mathrm{H}(\sigma, \bar{p}, \beta, C)}} \mathrm{d} \beta,
$$

Clearly, A.36 refers to the case where irreversibility is automatically fulfilled. On the contrary, one has to modify the construction as in case 2 on page 19 where a construction that accounts for irreversibility is investigated and explored.

It is worth noting that the initial width $D$ of the localization is greater for the $E-P-D$ response compared to the $E-D$ response, since $\mathrm{H}(\sigma, \bar{p}, \alpha, C) \geq \mathrm{H}(\sigma, 0, \alpha, C)$.

$E-P-P D$ response. In this case, when damage is triggered, plasticity must continue to evolve since the damage profile, constructed by assuming no singular points, violates the plastic criterion. That is, $\alpha_{\sigma}(x)$ in A.36 is such that $\sigma_{\mathrm{P}}(\alpha)<0$ somewhere. Clearly, as already discussed, the first point where the plastic criterion is attained is the center of the localization zone. Then, a candidate solution is to take the center of the localization zone as a singular point where plasticity localizes. The passages that follows are the same as in Appendix A.1 except for the presence of an initial uniform accumulated plastic strain in the governing equations which slightly modifies the results. The center of the localization zone $\bar{x}$ becomes a singular point, $J(\boldsymbol{\xi})=\{\bar{x}\}$, and the maximum damage level, attained in $\bar{x}$, is dictated for a given stress by A.25. In the region $J(\boldsymbol{\xi}) \backslash\{\bar{x}\}$ the damage profile is obtained from A.6 , leading to the implicit definition

$$
x-\bar{x}=\sqrt{\mathrm{w}_{1}} \ell \int_{\alpha}^{\bar{\alpha}(\sigma)} \frac{1}{\sqrt{\mathrm{H}(\sigma, \bar{p}, \beta, C)}} \mathrm{d} \beta,
$$




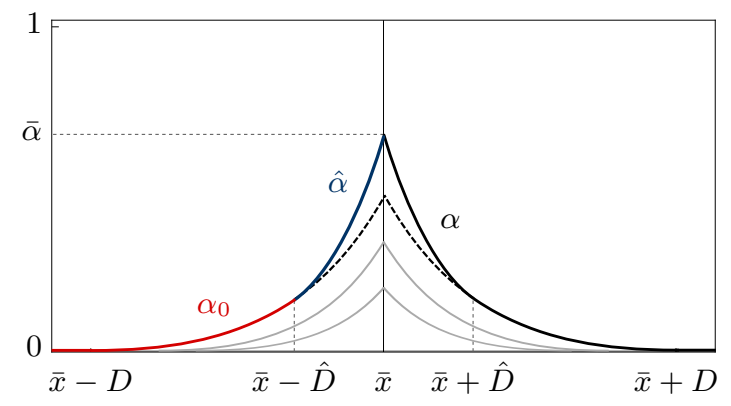

Figure A.14: The solid, black, thick curve represents the modified construction that satisfies irreversibility. The solid red branch is the profile which does not evolve because subjected to an elastic unloading while the blue branch represents an evolving profile. The solid gray curves represent the damage profiles at previous time instants. The damage derivative jumps at the tip of the profiles from the very beginning of the localization evolution.

where $\mathrm{H}(\sigma, \bar{p}, \beta, C)$ is the same function and has the same meaning as A.35. By the same line of reasoning in Appendix A.1 for the $E-D-P D$ case, the damage profile suffers in $\bar{x}$ a jump in its derivative, whose amplitude is given in A.27 and where coefficient 19 of the accumulated plastic strain Dirac measure is still given by A.28). In case where during the localization process irreversibility is not satisfied, the same changes in the construction of the damage profile for the $E-D-P D$ case have to be adopted, as schematically shown in Fig. A.14.

The damage profiles, corresponding to the constitutive models of Tab. 3 and used to determine the global responses in Sec. 5, have been built with the previous analytical constructions. The case of the $E-D-P D$ model is reported in Fig. A.15.

\section{Acknowledgement}

CM and SV express their sincere gratitude to Professor R.C. Batra for his generous advice, inspiring guidance and encouragement during the first years of their research activities. RA wishes to acknowledge the financial support of the MATHTECH-CNR-INdAM project.

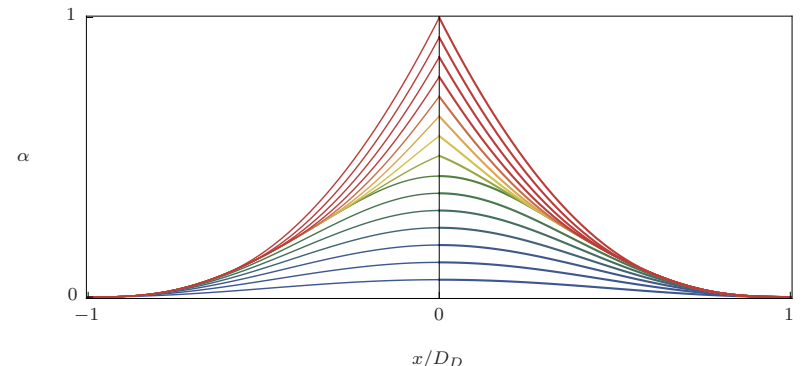

(a)

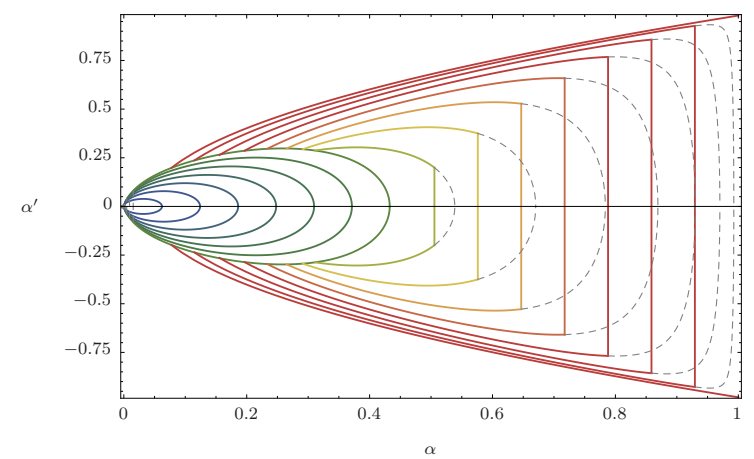

(b)

Figure A.15: Damage profiles (a) and the respective phase diagrams (b) of the $E-D-P D$ model obtained for different values of the stress.

\section{References}

[1] R. Alessi. Variational Approach to Fracture Mechanics with Plasticity. PhD thesis, Sapienza-Università di Roma/École Polytechnique, jul 2013.

[2] R. Alessi. Energetic formulation for rate-independent processes: remarks on discontinuous evolutions with a simple example. Acta Mechanica, 227(10):2805-2829, 2016.

[3] R. Alessi and D. Bernardini. Analysis of localization phenomena in Shape Memory Alloys bars by a variational approach. International Journal of Solids and Structures, 73-74:113-133, jun 2015.

[4] R. Alessi and F. Freddi. Phase-field modelling of failure in hybrid laminates. Composite Structures, Submitted, 2017.

[5] R. Alessi, J.-J. Marigo, and S. Vidoli. Gradient Damage Models Coupled with Plasticity and Nucleation of Cohesive Cracks. Archive for Rational Mechanics and Analysis, 214(2):575-615, jun 2014.

[6] R. Alessi, J.-J. Marigo, and S. Vidoli. Gradient damage models coupled with plasticity: Variational formulation and main properties. Mechanics of Materials, 80(Part B):351-367, 2015.

[7] R. Alessi and K. Pham. Variational formulation and stability analysis of a three dimensional superelastic model for shape memory alloys. Journal of the Mechanics and Physics of Solids, $87: 150-176$, feb 2016

[8] E. L. Allgower and K. Georg. Introduction to Numerical Continuation Methods. aug 2003.

[9] M. Alnæs, J. Blechta, J. Hake, A. Johansson, B. Kehlet, A. Logg, C. Richardson, J. Ring, M. E. Rognes, and G. N. Wells. The FEniCS Project Version 1.5, dec 2015.

[10] M. Ambati, T. Gerasimov, and L. De Lorenzis. Phase-field modeling of ductile fracture. Computational Mechanics, 55(5):10171040, apr 2015.

[11] M. Ambati, R. Kruse, and L. De Lorenzis. A phase-field model for ductile fracture at finite strains and its experimental verification. Computational Mechanics, 57(1):149-167, nov 2015.

[12] L. Ambrosio, A. Lemenant, and G. Royer-Carfagni. A Vari- 
ational Model for Plastic Slip and Its Regularization via $\Gamma$ Convergence. Journal of Elasticity, 110(2):201-235, feb 2013.

13] H. Amor, J.-J. Marigo, and C. Maurini. Regularized formulation of the variational brittle fracture with unilateral contact: Numerical experiments. Journal of the Mechanics and Physics of Solids, 57(8):1209-1229, aug 2009.

[14] S. Balay, S. Abhyankar, M. Adams, J. Brown, P. Brune, K. Buschelman, L. Dalcin, V. Eijkhout, W. Gropp, D. Kaushik, M. Knepley, L. C. McInnes, K. Rupp, B. Smith, S. Zampini, and H. Zhang. PETSc Users Manual. Technical Report ANL-95/11 - Revision 3.6, Argonne National Laboratory, 2015.

15] S. Balay, S. Abhyankar, M. Adams, J. Brown, P. Brune, K. Buschelman, L. Dalcin, V. Eijkhout, W. Gropp, D. Kaushik, M. Knepley, L. C. McInnes, K. Rupp, B. Smith, S. Zampini, and H. Zhang. PETSc Web page. http://www.mcs.anl.gov/petsc, 2015 .

[16] S. Balay, W. D. Gropp, L. C. McInnes, and B. F. Smith. Efficient Management of Parallelism in Object Oriented Numerical Software Libraries. In E. Arge, A. M. Bruaset, and H. P. Langtangen, editors, Modern Software Tools in Scientific Computing, pages 163-202. Birkhäuser Press, 1997.

[17] G. I. Barenblatt. The mathematical theory of equilibrium of cracks in brittle fracture. Advances in Applied Mechanics, 7:55129, 1962 .

[18] A. A. Benzerga and J. Leblond. Ductile fracture by void growth to coalescence. In Advances in Applied Mechanics, volume 44, pages 169-305. 2010.

[19] J. Besson. Continuum Models of Ductile Fracture: A Review, volume 19. apr 2009.

[20] M. J. Borden, T. J. R. Hughes, C. M. Landis, A. Anvari, and I. J. Lee. A phase-field formulation for fracture in ductile materials: Finite deformation balance law derivation, plastic degradation, and stress triaxiality effects. Computer Methods in Applied Mechanics and Engineering, 312:130-166, 2016.

21] B. Bourdin. Une méthode variationnelle en mécanique de la rupture, théorie et applications numériques (A Variational Method for Brittle Fracture, Theory and Numerical Implementation). PhD thesis, Université Paris Nord, Institut Galilée, France, 1998.

[22] B. Bourdin. Numerical implementation of the variational formulation for quasi-static brittle fracture. Interfaces and Free Boundaries, 9(3):411-430, 2007.

[23] B. Bourdin, G. A. Francfort, and J.-J. Marigo. Numerical experiments in revisited brittle fracture. Journal of the Mechanics and Physics of Solids, 48(4):797-826, apr 2000.

[24] B. Bourdin, G. A. Francfort, and J.-J. Marigo. The Variational Approach to Fracture. Journal of Elasticity, 91(1):5-148, mar 2008.

[25] B. Bourdin, J.-J. Marigo, C. Maurini, and P. Sicsic. Morphogenesis and Propagation of Complex Cracks Induced by Thermal Shocks. Physical Review Letters, 112(1):014301, jan 2014.

[26] A. Braides. Minimizing Movements. pages 91-101. Springer International Publishing, 2014.

[27] C. Comi. Computational modelling of gradient-enhanced damage in quasi-brittle materials. Mechanics of Cohesive-frictional Materials, 4(1):17-36, 1999

[28] C. Comi and U. Perego. Fracture energy based bi-dissipative damage model for concrete. International Journal of Solids and Structures, 38(36-37):6427-6454, 2001.

[29] S. Conti, M. Focardi, and F. Iurlano. Phase field approximation of cohesive fracture models. Annales de l'Institut Henri Poincare (C) Non Linear Analysis, 33(4):1033-1067, 2016.

[30] V. Crismale. Globally stable quasistatic evolution for a coupled elastoplastic-damage model. ESAIM: Control, Optimisation and Calculus of Variations, Accepted Paper, 2015.

[31] V. Crismale and G. Lazzaroni. Viscous approximation of quasistatic evolutions for a coupled elastoplastic-damage model Calculus of Variations and Partial Differential Equations, 55(1):17, jan 2016

[32] V. Crismale, G. Lazzaroni, and G. Orlando. Cohesive fracture with irreversibility: quasistatic evolution for a model subject to fatigue. Preprint SISSA;40/2016/MATE, 2016.

[33] G. Dal Maso, A. De Simone, and M. G. Mora. Quasistatic Evolution Problems for Linearly Elastic-Perfectly Plastic Materials. Archive for Rational Mechanics and Analysis, 180(2):237-291, feb 2006 .

[34] G. Dal Maso, G. Orlando, and R. Toader. Fracture models for elasto-plastic materials as limits of gradient damage models coupled with plasticity: the antiplane case. Calc. Var. Partial Differential Equations, 55(3):45, 2016.

[35] D. Dugdale. Yielding of steel sheets containing slits. Journal of Mechanics Physics of Solids, 8:100-104, may 1960.

[36] L. C. Evans and R. F. Gariepy. Measure Theory and Fine Properties of Functions. 1991

[37] G. A. Francfort, A. Giacomini, and J.-J. Marigo. A case study for uniqueness of elasto-plastic evolutions: The bi-axial test. Journal de Mathématiques Pures et Appliquées, 105(2):198227, feb 2016.

[38] G. A. Francfort, A. Giacomini, and J.-J. Marigo. The elastoplastic exquisite corpse: A Suquet legacy. Journal of the Mechanics and Physics of Solids, feb 2016.

[39] F. Freddi and F. Iurlano. Numerical insight of a variational smeared approach to cohesive fracture. Journal of the Mechanics and Physics of Solids, 98:156-171, 2017.

[40] F. Freddi and G. Royer-Carfagni. Regularized variational theories of fracture: A unified approach. Journal of the Mechanics and Physics of Solids, 58(8):1154-1174, aug 2010.

[41] F. Freddi and G. Royer-Carfagni. Phase-field slip-line theory of plasticity. Journal of the Mechanics and Physics of Solids, 2016

[42] A. A. Griffith. The phenomena of rupture and flow in solids. Philosophical Transactions of the Royal Society of London. Series A, Containing Papers of a Mathematical or Physical Character, 221:pp. 163-198, 1920.

[43] A. L. Gurson. Continuum Theory of Ductile Rupture by Void Nucleation and Growth: Part I - Yield Criteria and Flow Rules for Porous Ductile Media. Journal of Engineering Materials and Technology, Transactions of the ASME, 99 Ser H(1):2-15, 1977.

[44] B. Halphen and Q. S. Nguyen. Generalized Standard Materials. Journal de Mécanique, 14(1):39-63, 1975.

[45] W. Han and B. D. Reddy. Plasticity. Mathematical theory and numerical analysis, volume Springer V of Interdisciplinary applied mathematics: Mechanics and materials. Springer, 1999.

[46] C. Kuhn, T. Noll, and R. Müller. On phase field modeling of ductile fracture. GAMM-Mitteilungen, 39(1):35-54, jun 2016.

[47] G. Lancioni. Modeling the Response of Tensile Steel Bars by Means of Incremental Energy Minimization. Journal of Elasticity, 121(1):25-54, feb 2015.

[48] G. Lancioni, T. Yalçinkaya, and A. Cocks. Energy-based nonlocal plasticity models for deformation patterning, localization and fracture. Proceedings of the Royal Society A: Mathematical, Physical and Engineering Science, 471(2180), jul 2015.

[49] A. A. León Baldelli, J. F. Babadjian, B. Bourdin, D. Henao, and C. Maurini. A variational model for fracture and debonding of thin films under in-plane loadings. Journal of the Mechanics and Physics of Solids, 70:320-348, oct 2014.

[50] A. Logg, K.-A. Mardal, and G. Wells. Automated Solution of Differential Equations by the Finite Element Method: The FEniCS Book. feb 2012

[51] J.-J. Marigo, C. Maurini, and K. Pham. An overview of the modelling of fracture by gradient damage models. Meccanica, 51(12):3107-3128, dec 2016

[52] C. Miehe, M. Hofacker, L.-M. Schänzel, and F. Aldakheel. Phase field modeling of fracture in multi-physics problems. Part II. Coupled brittle-to-ductile failure criteria and crack propagation in thermo-elastic-plastic solids. Computer Methods in Applied Mechanics and Engineering, 294:486-522, sep 2015.

[53] C. Miehe, S. Teichtmeister, and F. Aldakheel. Phase-field modelling of ductile fracture: a variational gradient-extended plasticity-damage theory and its micromorphic regularization. Philosophical transactions. Series A, Mathematical, physical, 
and engineering sciences, 374(2066), apr 2016.

[54] A. Mielke. A Mathematical Framework for Generalized Standard Materials in the Rate-Independent Case. In R. Helmig, A. Mielke, and B. Wohlmuth, editors, Multifield Problems in Solid and Fluid Mechanics, volume 28 of Lecture Notes in Applied and Computational Mechanics, pages 399-428. Springer Berlin / Heidelberg, 2006.

[55] A. Mielke and T. Roubíček. Rate-Independent Systems: Theory and Application. Springer, 2015.

[56] H. Petryk. Incremental energy minimization in dissipative solids. Comptes Rendus Mecanique, 331(7):469-474, jul 2003.

57] K. Pham, H. Amor, J.-J. Marigo, and C. Maurini. Gradient Damage Models and Their Use to Approximate Brittle Fracture. International Journal of Damage Mechanics, 20(4):618$652,2011$.

[58] K. Pham and J.-J. Marigo. Approche variationnelle de l'endommagement : II. Les modèles à gradient. Comptes Rendus Mecanique, 338(4):199-206, 2010.

[59] K. Pham and J.-J. Marigo. Stability of Homogeneous States with Gradient Damage Models: Size Effects and Shape Effects in the Three-Dimensional Setting. Journal of Elasticity, 110(1):63-93, apr 2012.

[60] K. Pham, J.-J. Marigo, and C. Maurini. The issues of the uniqueness and the stability of the homogeneous response in uniaxial tests with gradient damage models. Journal of the Mechanics and Physics of Solids, 59(6):1163-1190, 2011.

[61] O. Rokoš, J. Zeman, and M. Jirásek. Localization analysis of an energy-based fourth-order gradient plasticity model. European Journal of Mechanics - A/Solids, 55:256-277, 2016.

[62] T. Roubíček and J. Valdman. Perfect plasticity with damage and healing at small strains, its modelling, analysis, and computer implementation. may 2015.

[63] G. Rousselier. Ductile fracture models and their potential in local approach of fracture. Nuclear Engineering and Design, 105(1):97-111, 1987.

[64] P. M. Suquet. Sur les équations de la plasticité: existence et régularité des solutions. Journal de Mécanique, 20:3-40, 1981.

[65] V. Tvergaard and A. Needleman. Analysis of the cup-cone fracture in a round tensile bar. Acta Metallurgica, 32(1):157-169, jan 1984.

[66] J. Ulloa, P. Rodríguez, and E. Samaniego. On the modeling of dissipative mechanisms in a ductile softening bar. Journal of Mechanics of Materials and Structures, 11(4):463-490, aug 2016. 